\title{
A variational formulation for higher order macroscopic traffic flow models: numerical investigation
}

\author{
G. Costeseque ${ }^{* * \dagger}$, J.P. Lebacque ${ }^{\dagger}$
}

September 17, 2013

\begin{abstract}
This paper deals with numerical methods providing semi-analytic solutions to a wide class of macroscopic traffic flow models for piecewise affine initial and boundary conditions. In a very recent paper, a variational principle has been proved for models of the Generic Second Order Modeling (GSOM) family, yielding an adequate framework for effective numerical methods. Any model of the GSOM family can be recast into its Lagrangian form as a Hamilton-Jacobi equation (HJ) for which the solution is interpreted as the position of vehicles. This solution can be computed thanks to Lax-Hopf like formulas and a generalization of the inf-morphism property. The efficiency of this computational method is illustrated through a numerical example and finally a discussion about future developments is provided.
\end{abstract}

Keywords: Traffic flow, Hamilton-Jacobi equation, Lax-Hopf algorithm, Lagrangian.

\section{Introduction}

\subsection{General background}

Macroscopic traffic flow modeling. In order to get a realistic estimation of the real-time traffic states on networks, traffic operators and managers need macroscopic traffic flow models. These models must be simple, robust, allowing to get solutions at a low computational cost. The main macroscopic models are based on conservation laws or hyperbolic systems (see [30, 19] for a review). The seminal LWR model (for Lighthill-Whitham and Richards) was proposed in [42, 51] as a single conservation law with unknown the vehicles density. This model based on a first order Partial Differential Equation (PDE) is very simple and robust but it fails to recapture some empirical features of traffic. In particular, it does not allow to take into account non-equilibrium traffic states mainly in congested situation. More sophisticated models referred to as higher order models were developed to encompass kinematic constraints of real vehicles or also the wide variety of driver behaviors, even at the macroscopic level. In this paper we deal with models of the Generic Second Order Modeling (GSOM) family. Even if these models are more complicated to deal with, they permit to reproduce traffic instabilities (such as the so-called stop-and-go waves, the hysteresis phenomenon or capacity drop) which move at the traffic speed and differ from kinematic waves [53] (see also [36] and references therein).

${ }^{*}$ Université Paris-Est, Ecole des Ponts ParisTech, CERMICS, 6 \& 8 avenue Blaise Pascal, Cité Descartes, Champs sur Marne, 77455 Marne la Vallée Cedex 2, France

${ }^{\dagger}$ Université Paris-Est, IFSTTAR, GRETTIA, 14-20 Boulevard Newton, Cité Descartes, Champs sur Marne, 77447 Marne la Vallée Cedex 2, France.

${ }^{*}$ Corresponding author, e-mail: costeseg@cermics.enpc.fr 
Traffic flow monitoring. Before the wide propagation of internet handsets, traffic monitoring has mainly been built on dedicated infrastructure which imply quite important installation and maintenance costs. Traffic flow monitoring and management has been deeply modified with the development of new technologies in mobile sensing aiming to provide a quite important quantity of floating car data. Traffic flow models are needed to be well suited such that managers could use both Eulerian and Lagrangian data for improving traffic state estimation. The term Eulerian refers to "classical" fixed equipment giving records of occupancy or flow of vehicles on a freeway section. This kind of measurements come from e.g. fixed inductive loop detectors, Radio Frequency Identification (RFID) transponders, radars or video cameras. By opposite, the term Lagrangian is used to characterize data coming from sensors which move within the measured field of interest. Lagrangian data are provided by on board mobile sensors such as Global Positioning Systems (GPS) or GPS-enabled smartphones.

Data assimilation. While the idea of monitoring traffic using mobile sensors appeared less than ten years ago with the popularization of the mobile internet devices, there exists a fast growing literature about how to integrate Lagrangian data into classical macroscopic traffic flow models. The process of incorporating Eulerian and Lagrangian data into a mathematical model to improve the modeling is called data estimation or equivalently inverse modeling. According to the major UC Berkeley field experiment named Mobile Century and then Mobile Millennium [54, it has been shown that even a $2 \%$ to $5 \%$ penetration rate of probe vehicles into the driver population, Lagrangian sensing provides sufficient and accurate data for estimating traffic velocity or density on highways [22, 23, 52]. Nevertheless, it has been demonstrated in 49 that the quality of estimation for higher-order traffic quantities including vehicles acceleration/deceleration, emission and fuel consumption rates is dramatically affected when the penetration rate of probe vehicles or the sampling frequency of the current mobile sensors decrease. However on board devices propose a real breakthrough in traffic monitoring by providing a very cheap and efficient way to collect traffic data.

\subsection{Motivation}

In order to improve traffic states estimation from Lagrangian data, we propose to deal with macroscopic traffic flow models of the GSOM family. As these models combine the simplicity of the LWR model with the dynamics of driver specific attributes, we are able to recapture more specific phenomenon with a higher accuracy. While methods of data assimilation have been only developed for first order models up to now, this work presents a new algorithm to reconstruct traffic states from both Eulerian and Lagrangian data. We take advantage of a very recent article [34] in which a variational principle has been proved for models of the GSOM family.

\subsection{Organization of the paper}

The rest of this paper is structured as follows. Section 2 presents more in detail the GSOM models and sheds a specific light on the LWR model which is widely used in traffic engineering. The variational principle for the GSOM models is briefly recalled in Section 3, Section 4 is devoted to the presentation of the main elements of our computational method. Finally, Section 5 proposes some numerical examples and a cross-comparison of our numerical method and a more classical one based on finite differences. 


\section{GSOM traffic flow models}

\subsection{Formulation of GSOM models}

In [35, 36], the authors introduce a general class of macroscopic traffic flow models called the Generic Second Order Models (GSOM) family. Any model of the GSOM family can be stated in conservation form as follows

$$
\begin{cases}\partial_{t} \rho+\partial_{x}(\rho v)=0 & \text { Conservation of vehicles, } \\ \partial_{t}(\rho I)+\partial_{x}(\rho v I)=\rho \varphi(I) & \text { Dynamics of the driver attribute } I, \\ v=\Im(\rho, I) & \text { Fundamental diagram, }\end{cases}
$$

where $\rho$ stands for the density of vehicles, $v$ for the flow speed (equal to the mean spatial velocity of vehicles), $x$ and $t$ for position and time. The variable $I$ is a specific driver attribute which can represent for example the driver aggressiveness, the driver destination or the vehicle class. The flow-density fundamental diagram (FD) is defined by

$$
\mathfrak{F}:(\rho, I) \mapsto \rho \mathfrak{I}(\rho, I) .
$$

Notice moreover that it was shown in [36] that the notions of Supply and Demand functions defined in [30, 31] for the classical LWR model could be extended to the GSOM family. The GSOM models admit two kinds of waves:

- Kinematic waves or 1-waves as in the seminal LWR model: a wave propagates density variations at speed $\nu=\partial_{\rho} \Im(\rho, I)$ while the driver attribute $I$ is continuous across such a wave.

- Contact discontinuities or 2-waves: a wave propagates variations of driver attribute $I$ at speed $\nu=\mathfrak{I}(\rho, I)$ while the flow speed is constant across such a wave.

\section{$2.2 \quad$ Examples}

The GSOM family recovers a wide range of existing models:

- The LWR model [42, 51] itself is simply a GSOM model with no specific driver attribute, expressed as follows

$$
\begin{cases}\partial_{t} \rho+\partial_{x}(\rho v)=0 & \text { Conservation of vehicles, } \\ v=\mathfrak{I}(\rho, x) & \text { Fundamental diagram. }\end{cases}
$$

The fundamental diagram (FD) for the LWR model states that traffic flow is always at an equilibrium state. It is commonly assumed that the flow is an increasing function of density between zero (corresponding to an empty section) and a critical density and then the flow decreases until the maximal density (corresponding to a bumper to bumper situation). However the FD shape is always a subject of debates and there exists a wide variety in the literature encompassing concave and triangular flow functions (see Figure 1 and also Chapter 3 of [19] for additional examples).

- The LWR model with bounded acceleration proposed in [32, 33, 39] is also a GSOM model in which the propagated driver attribute is simply the speed of vehicles.

- The ARZ model (standing for Aw, Rascle [1] and Zhang [53]) for which the driver attribute is taken as $I=v-V_{e}(\rho)$ that is $\mathfrak{I}(\rho, I)=I+V_{e}(\rho)$.

- Multi-commodity models (multi-class, multi-lanes) of Jin and Zhang [26], Bagnerini and Rascle [4] or Herty, Kirchner, Moutari and Rascle [24]. It encompasses also the model of Klar, Greenberg and Rascle [27. 

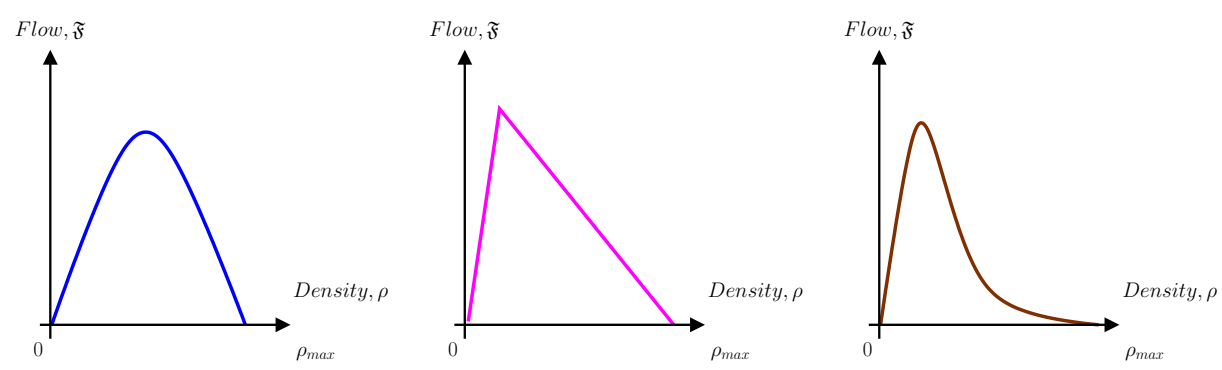

Figure 1: Illustrations of some flow functions $\mathfrak{F}$ for the LWR model: Greenshields (left), triangular (center) and exponential (right).

- The Colombo 1-phase model deduced in [36] from the 2-phase model of Colombo [9]. In this case, the driver attribute $I$ is a scalar which is non-trivial in congested situation. In fluid area, the model follows the classical LWR model.

- The stochastic GSOM model of Khoshyaran and Lebacque [28. The driver attribute $I$ is a random variable depending on the vehicle index $N$ and on the random event $\omega$ such that $I=I(N, t, \omega)$. The random perturbations do not affect the vehicle dynamics but affect the driver perception and its behaviour.

The interested reader is referred to [34] and references therein for more details on examples.

\section{Variational principles in traffic flow modeling}

In traffic flow literature, the variational formulation was first conjectured by Newell in [47] for the LWR first-order traffic flow model. It was then properly established and generalized by Daganzo in 13, 14, 15, 16. In this section, we first present the three dimensional representation of traffic flow or the so-called Moskowitz function [46, 44] and the Hamilton-Jacobi formulation of the LWR model (see also [29] and references therein). The numerical methods for this scalar case are then described. Finally we introduce the variational formulation of the GSOM family lead by the ideas developed for the LWR model.

\subsection{Variational formulation of the LWR model}

If one assumes that traffic flow on a section could be represented by a continuum of vehicles, we can define the cumulative vehicles count (CVC) by interpolating the count of each passing vehicle at a location as a continuous function of space and time $N(x, t)$ which can be represented as a surface in space $t-x-n$ often referred to as the Moskowitz surface. The function $N(x, t)$ allows to compute useful traffic variables such as

- the individual trajectory of vehicle $n_{j}$ by projection on the Eulerian plane $t-x$.

- the traffic flow $\mathfrak{f}:=\partial_{t} N$ at a location $x_{i}$ or the travel time between two locations $x_{i}$ and $x_{i+1}$ by projection on the Lagrangian plane $t-n$.

- the vehicles density $\rho=-\partial_{x} N$ at time $t_{i}$ or the traveled distance by a vehicle between times $t_{i}$ and $t_{i+1}$ by projection on the plane $x-n$.

If the density $\rho$ solves the LWR model (2.2) then it is well known that the function $N$ solves the following Hamilton-Jacobi equation

$$
\partial_{t} N-\mathfrak{F}\left(-\partial_{x} N\right)=0 .
$$


Under suitable assumptions, the (viscosity) solution to the HJ equation (3.3) could be determined thanks to a variational formulation, known as Lax-Hopf formula in the mathematics literature [18]. There exist different methods to obtain the variational formulation of this problem, including calculus of variations and viability theory.

Let us study the variations of the function $N$ from the initial trajectory data $\mathcal{J}$ which synthesizes the initial conditions and the boundaries conditions in the case of a finite section.

\subsubsection{Eulerian setting}

Consider the Eulerian frame with $t-x$ fixed. A fictitious observer referenced by its position $X(t)$ moving in the traffic flow at a given speed $\dot{X}(t)$ is overtaken by a number of vehicles given by

$$
\mathcal{M}(\dot{X}(t))=\sup _{\rho}[\mathfrak{F}(\rho(t, X(t)))-\rho(t, X(t)) \dot{X}(t)],
$$

with $\mathfrak{F}$ the flow, linked to the density $\rho$ by the fundamental diagram. The evolution of the flow through a trajectory $X(t)$ is then described by the function $\mathcal{M}(\dot{X})$. This function refers to the relative capacity in traffic and it matches the Lagrangian of the system. Thus knowing the function $N$ at a point $A$ of $\mathcal{J}$, it is possible to compute the value of $N$ at a point $B$ by estimating $\mathcal{M}$ along the path $\mathcal{P}_{A B}$ followed by the observer from $A$ to $B$

$$
\Delta N_{A B}=\int_{\mathcal{P}_{A B}}\{\mathfrak{F}(\rho(t, X(t)))-\rho(t, X(t)) \dot{X}(t)\} d t
$$

As the differential of $N(t, x)$ is a total and exact differential, it does not depend on the followed path. However $\Delta N_{A B}$ depends on the density and the flow of the chosen path which are unknown. The solution $N$ between $A$ and $B$ is computed by the following dynamic programming equation which states that the variation of $N$ comply to the Maupertuis minimal action principle

$$
\begin{aligned}
N\left(T, x_{T}\right)= & \min _{\dot{X}(.),\left(t_{0}, x_{0}\right)} \int_{t_{0}}^{T} \mathcal{M}(\dot{X}(\tau)) d \tau+N\left(t_{0}, x_{0}\right), \\
& \mid \begin{array}{l}
X(.) \in \mathcal{U} \\
X\left(t_{0}\right)=x_{0}, \quad X(T)=x_{T} \\
\left(t_{0}, x_{0}\right) \in \mathcal{J}
\end{array}
\end{aligned}
$$

where $\mathcal{U}$ denotes the set of admissible paths (smooth enough) connecting the initial locus $\mathcal{J}$ to the "target" final point $\left(T, x_{T}\right)$.

The conditions for the problem (3.4) to solve (3.3) are the following ones [13, 14]:

- The flow-density fundamental diagram $\mathfrak{F}(\rho)$ is concave with respect to $\rho$ such that the Hamiltonian defined as $H(t, X, \rho):=\mathcal{M}(\dot{X})+\rho \dot{X}=\mathfrak{F}(\rho(t, X(t)))$ is itself concave w.r.t. its argument $\rho$.

- The optimal path maximizes the relative capacity. On the optimal trajectory $X^{*}$, we define the maximal relative capacity (or the maximal overflow according to Daganzo) defines the path cost and matches the Legendre transform of $\mathfrak{F}$

$$
\mathfrak{M}=\mathcal{M}\left(\dot{X}^{*}\right)=\sup _{\rho}\left[\mathfrak{F}(\rho)-\rho \dot{X}^{*}\right] .
$$

- As solutions of (3.4) must solve the LWR model (2.2), the admissible speeds need to comply to $\dot{X}=\partial_{\rho} \mathfrak{F}$ and be equal to the speed of kinematic waves for which the (constant) density solves the LWR model (2.2).

- The optimal trajectory leading to the value $N$ at every point $(t, x)$ is a straight line coming from the initial and boundaries conditions $\mathcal{J}$. 
- The solutions agree the boundaries conditions $\mathcal{J}$.

In the particular case of a triangular flow-density fundamental diagram, there are only two kinds of waves that can reach the target point: either shock wave moving forward from point $x_{u}$ at speed $u$ or rarefaction wave moving backward from position $x_{w}$ at speed $w$ (see Figure 2). Then we can recover that the solution is given by

$$
N(t, x)=\min \left[N\left(t-\frac{x-x_{u}}{u}, x_{u}\right), N\left(t-\frac{x-x_{w}}{w}, x_{w}\right)+\rho_{\max }\left(x_{w}-x\right)\right],
$$

where $u$ and $w$ are respectively the free flow speed and the congested speed. This formulation was stated by Newell [47] and properly proved by Daganzo [13, 14].
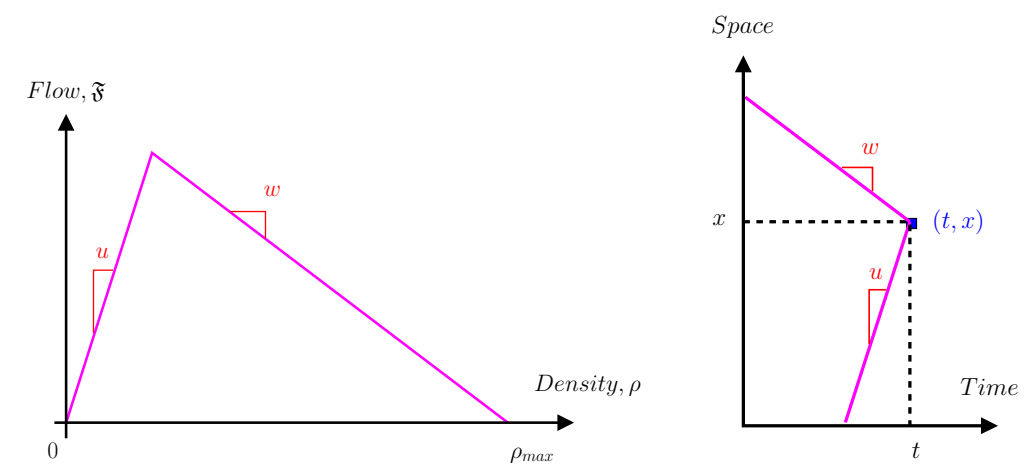

Figure 2: Optimal paths candidates with a triangular flow function $\mathfrak{F}$ for LWR model.

\subsubsection{Lagrangian setting}

Consider the Lagrangian framework with $t-n$ fixed (see also [40] for a presentation of Lagrangian setting in traffic flow). The function $X(t, n)$ represents the trajectory of vehicle $n$ when it is an integer. The initial and boundary conditions $\mathcal{J}$ are given respectively by the trajectory of the first vehicle, by the times when the other vehicles enter the section and by the initial positions of vehicles. Let us define the spacing $r:=1 / \rho$ and we set the speed-spacing fundamental diagram $\mathcal{V}$ as follows

$$
\mathcal{V}: r \mapsto \mathfrak{I}(1 / r) .
$$

As in the Eulerian setting, it is well known that the function $X(t, n)$ solves the following Hamilton-Jacobi equation

$$
\partial_{t} X-\mathcal{V}\left(-\partial_{n} X\right)=0
$$

Notice that from a mathematical point of view it was rigorously established in [20] that the viscosity solutions of (3.3) and (3.6) are strictly equivalent.

The study of the evolution of $X$ between points $A$ and $B$ in the plane $t-n$ is identical to the previous one in the Eulerian setting. We define the Lagrangian such that

$$
\mathcal{M}(\dot{N}(t))=\sup _{r}[\mathcal{V}(r(t, N(t)))-r(t, N(t)) \dot{N}(t)] .
$$

The functional expression of the associate problem is given by (3.7) which is the dual formulation of (3.4)

$$
\begin{aligned}
X\left(T, n_{T}\right)= & \min _{\dot{N}(.),\left(t_{0}, n_{0}\right)} \int_{t_{0}}^{T} \mathcal{M}(\dot{N}(\tau)) d \tau+X\left(t_{0}, n_{0}\right) \\
& \mid \begin{array}{l}
N(.) \in \mathcal{N} \\
N\left(t_{0}\right)=n_{0}, \quad N(T)=n_{T} \\
\left(t_{0}, n_{0}\right) \in \mathcal{J}
\end{array}
\end{aligned}
$$


where $\mathcal{N}$ denotes the set of admissible paths (smooth enough) connecting the initial locus $\mathcal{J}$ to the "target" final point $\left(T, n_{T}\right)$.

The conditions for the problem (3.7) to solve (3.6) are similar to the ones for the Eulerian case. We then can consider a restricted number of possible paths in $t-n$. Especially, we have that

- The fist condition giving that Hamiltonian is maximal conduces to set $\mathcal{M}(\dot{N})$ as the Legendre transform of $\mathcal{V}$ (the speed-spacing FD) which is concave w.r.t. $r$.

- The Hamilton-Jacobi equation is satisfied along the optimal trajectory $N^{*}(t)$ and

$$
\dot{N}=\partial_{r} \mathcal{V} \quad \text { and } \quad \dot{r}=-\partial_{n} \mathcal{V} \text {. }
$$

- The optimal paths are straight lines which slopes are $\partial_{r} \mathcal{V}$ and they satisfy the boundary conditions $\mathcal{J}$.

In the particular case of the piecewise affine speed-spacing fundamental diagram (see Figure 3) corresponding to the triangular flow-density fundamental diagram, the formula (3.7) becomes

$$
X(t, n)=\min \left[X\left(t_{0}, n\right)+u\left(t-t_{0}\right), X\left(t_{0}, n+w \rho_{\max }\left(t-t_{0}\right)\right)+w\left(t-t_{0}\right)\right] .
$$

We recover the microscopic follow-the-leader model of Newell [48, expressed in Lagrangian coordinates. This model states that the trajectory of a vehicle is given by the minimum between the vehicle trajectory at the free speed $u$ (under-critical situation) and a translation of the leader vehicle (over-critical situation).
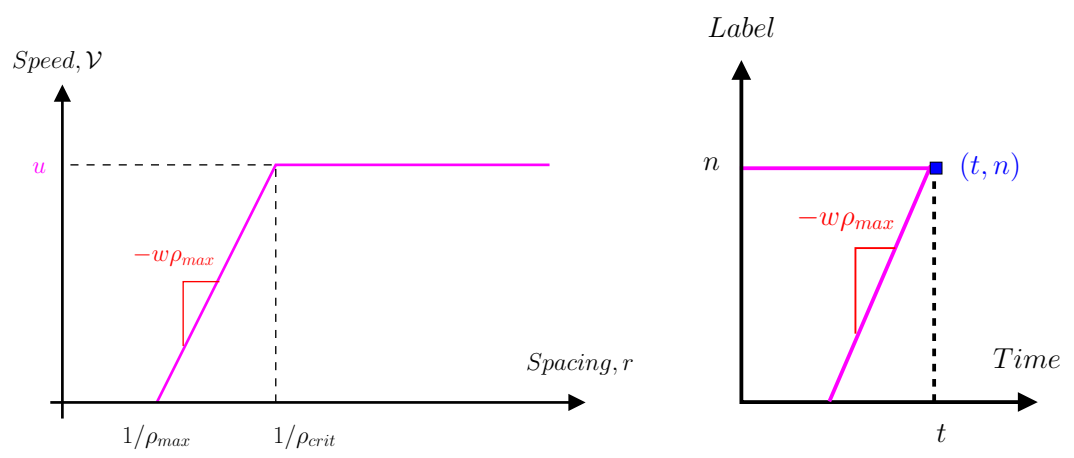

Figure 3: Optimal paths candidates with a triangular flow function $\mathcal{V}$ for LWR model.

\subsection{Computational aspects for the LWR model}

The LWR model is built on a first order scalar conservation law for which there generally does not exists a unique and explicit solution for infinite time horizon due to discontinuities as shock waves for example. However the physical meaningful solution known as the entropy solution can be computed by numerical non-variational methods that encompass:

- finite volume schemes such as the Godunov scheme which was translated into traffic in [12, 30, 31. These schemes need a computational grid and except for very specific cases, they are non-exact.

- the wave tracking method [21, 25] which simplifies the calculations by only following the meaningful kinematic waves that is shocks or rarefaction waves. However this method is event-based since it needs to evaluate each time when two kinematic waves meet and in practice it is restricted to piecewise linear flow-density fundamental diagrams (and piecewise initial and boundary conditions) for dealing with a finite number of wave speeds. 


\subsubsection{Motivation of Lax-Hopf algorithms}

The variational formulation of the LWR model has found many interesting applications since it was discovered. Among others, it allows to improve modeling flexibility e.g. taking into account moving bottlenecks [14, 15] and it provides accurate algorithms for numerical treatment and data assimilation [7, 8, 20, 45].

Up to our best knowledge, existing numerical procedures relying on variational formulation of the LWR model use the seminal paper of Claudel and Bayen [6] which proposes a semiexplicit form of the solution to Hamilton-Jacobi equations using control techniques based on viability theory [2, 3. They define the proper lower semi-continuous solution (in the BarronJensen / Frankowska sense) to the Hamilton-Jacobi equation (3.3) with concave flow-density fundamental diagrams. Moreover this solution satisfies an initial condition, upstream and downstream boundary conditions (Eulerian data) and additional internal boundary conditions to take into account Lagrangian data.

For this aim, Claudel and Bayen [6] use a generalized Lax-Hopf formula (which allows to easily prove the well-posedness of the problem with internal boundary data) and the inf-morphism property [2, 3] to compute the solution by taking the infimum of all solutions associated with simpler sub-problems. It follows computational methods [7] that are commonly referenced as "grid free" schemes in the sense that they do not need a two-dimensional discretization of the domain as opposed to classical finite difference schemes. Indeed Lax-Hopf algorithm allows to avoid the use of a computational grid (whatever its shape). The inf-morphism property goes back to sup-morphism property obtained in [2, 3] from the viability theory. It allows to divide the general problem involving multiple value conditions into several independent and simpler problems involving a single value condition. Numerically, the inf-morphism property permits a low computational cost thanks to the increasing power of parallel computing.

Unlike dynamic programming methods [13], Lax-Hopf algorithms have been proved to be exact in general cases of concave flow-density fundamental diagrams (or convex Hamiltonians) [6].

\subsubsection{Eulerian setting}

Thanks to the Lax-Hopf algorithm [6] which is not event-based and does not use a grid of the space-time domain, the authors in [7, 45] solve the LWR model restated under its Hamilton-Jacobi formulation. They can deal with any concave fundamental diagram (essential to properly define the convex transform) and any piecewise constant initial and boundary conditions in terms of density, that is equivalent to consider any piecewise affine (PWA) initial and boundary conditions for the Moskowitz function. Eventually Lagrangian data could be assimilated as internal boundary conditions. Notice that the flow-density function (and its convex transform) could be non-differentiable at a finite number of points. That will only modify the value of the computed solution at these points where the exact solution is discontinuous anyway and it is not defined.

The method is exact and allows to compute exactly the derivative of the (unique viscosity) solution of the HJ equation which matches the (unique entropy) solution of the LWR model. Some elements of comparison are given in [45. between dynamic programming, Lax-Hopf algorithm and more classical methods such as Godunov scheme and wave tracking algorithm. Globally, Lax-Hopf algorithm seems to provide the best method compared to the others because it gives the exact solutions at a weaker computation cost than the other methods.

\subsubsection{Lagrangian setting}

The proposed algorithm in [45] has been extended to the Lagrangian counterpart of the problem in [20]. This paper underlines that Lagrangian coordinates have the advantage of providing information associated with a given vehicle. Moreover it is worth noticing that in the Eulerian setting, one need to take into account forward and backward fans for determining the 
domain of influence of any value condition. In the Lagrangian setting, one needs to look after the forward fan, since the wave propagation speeds are necessarily non negative. Indeed the speed-spacing fundamental diagram is monotonically increasing. This implies that any value condition can only influence the solution $\mathcal{X}$ for larger labels $N$. From a traffic point of view, it means that the perturbation generated by a vehicle can only affect vehicles that come behind. As the problem in Lagrangian coordinates involves a similar Hamilton-Jacobi equation to the one in Eulerian coordinates, the authors in 20 provide semi-analytical solutions for a triangular flow-density FD and for piecewise affine initial and boundary conditions. The upstream and downstream boundary conditions refer to the trajectories of the first and last vehicles which are within the scope. Internal conditions such as Eulerian data from fixed sensors can also be taken into consideration. The comparison between the numerical results and the real observations carried out from the Mobile Century field experiment [54] shows the accuracy of the algorithm.

\subsection{Variational formulation of the GSOM family}

The ability of the LWR model to reproduce certain complicated macroscopic traffic phenomena such that capacity drop or stop-and-go waves is known to be weak. For this reason we have focused on developing variational methods for GSOM models. Guided by the ideas developed for the LWR model, variational formulations have been recently developed and proved for models of the GSOM family in both Eulerian [41] and Lagrangian frameworks [34].

\subsubsection{Eulerian setting}

In [41, the authors prove the existence of a variational formulation of a class of models from the GSOM family. Unlike the paper of Lebacque and Khoshyaran [34] which will be discussed just after, these models are expressed from the Eulerian viewpoint as a system of two conservation laws (the first one for the conservation of vehicles and the second one for the behavioral motion of traffic flow around an equilibrium state)

$$
\begin{cases}\partial_{t} \rho+\partial_{x} f(\rho, s)=0 & \text { Conservation of vehicles } \\ \partial_{t} s+\partial_{x} g(\rho, s)=0 & \text { Dynamics around the equilibrium. }\end{cases}
$$

The first conservation law is obviously satisfied by the vehicles density $\rho$ and the corresponding flux $f$ is the product of density times speed $f(\rho, v)=\rho v$. The second conservation law is satisfied by a variable $s$ (a non-equilibrium measure) which can be replaced by $s=\rho I$ yielding to the GSOM family without source terms. The corresponding flux $g$ needs to comply some specific conditions to ensure that the wave speed is less or equal to traffic speed. The authors assume that $f$ is concave with respect to $\rho$ for any $s$ and $g$ is convex in $s$ for any $\rho$.

The paper [41] particularly deals with the generic formulation of GSOM models without the source term that is $\varphi(I)=0$. This is equivalent to say that the driver attribute $I$ is invariant along vehicles trajectories which seems to be corroborated for instance by the work of Duret et al. 17] on the NGSIM I-80 trajectories data-set (in congested situation). For the models (3.10), the authors show in [41] a variational formulation based on Lax-Hopf like formulas (i.e. the value function at a point is equal to the minimum of the sum of the initial value and a trajectory cost over every possible paths from initial and boundary data reaching the final point) for both $N_{\rho}$ and $N_{s}$ defined as the cumulative quantity of respectively $\rho$ and $s$.

When taking into account a non trivial relaxation term $(\varphi(I) \neq 0)$, the authors assume that the problem reduces to solving a LWR model in large time because the relaxation term induces an exponential decay in time of the difference between (3.10) and a regular LWR model. 


\subsubsection{Lagrangian setting}

In [34, the authors prove the existence of a variational principle for the GSOM models family. In a first step, the article gives the Hamilton-Jacobi formulation of GSOM models. Consider one GSOM model described by (3.10) and let us express it under its Lagrangian form with $r$ the spacing and $N$ the vehicle label

$$
\begin{cases}\partial_{t} r+\partial_{N} v=0 & \text { Conservation of vehicles, } \\ \partial_{t} I=\varphi(N, I, t) & \text { Dynamics of the driver attribute } I, \\ v=\mathcal{V}(r, I)=\Im(1 / r, I) & \text { Fundamental diagram. }\end{cases}
$$

Considering the position $\mathcal{X}(N, t)=\int_{-\infty}^{t} v(N, \tau) d \tau$, we obviously have that

$$
v=\partial_{t} \mathcal{X} \text { and } r=-\partial_{N} \mathcal{X} .
$$

Hence the system (3.11) could be written as a Hamilton-Jacobi equation satisfied by $\mathcal{X}$ :

$$
\partial_{t} \mathcal{X}-\mathcal{W}\left(N,-\partial_{N} \mathcal{X}, t\right)=0
$$

where $\mathcal{W}$ denotes the speed-spacing fundamental diagram of vehicle $N$ at time $t$. It is defined such that

$$
\mathcal{W}(N, r, t):=\mathcal{V}(r, I(N, t))=\mathfrak{I}(1 / r, I(N, t)),
$$

where the driver attribute $I(N, t)$ solves the following nonlinear first order ODE

$$
\mid \begin{aligned}
& \partial_{t} I(N, t)=\varphi(N, I, t), \\
& I(N, 0)=i_{0}(N), \quad \text { for any } \quad N .
\end{aligned}
$$

By classical results on optimal control problems i.e. dynamic programming on HamiltonJacobi-Bellman equations, we can check that:

$$
\begin{aligned}
\mathcal{X}\left(N_{T}, T\right)=\min _{u,\left(N_{0}, t_{0}\right)} \int_{t_{0}}^{T} \mathcal{M}(N, u, t) d t+\xi\left(N_{0}, t_{0}\right), \\
\qquad \begin{array}{l}
\dot{N}=u \\
N\left(t_{0}\right)=N_{0}, \quad N(T)=N_{T} \\
\left(N_{0}, t_{0}\right) \in \mathcal{J}
\end{array}
\end{aligned}
$$

where $\mathcal{M}$ is the Legendre-Fenchel transform of $\mathcal{W}$ according to its second variable (see Figure 4 for an illustration of the Legendre-Fenchel transform), defined such that

$$
\mathcal{M}(N, u, t)=\sup _{r \in \mathbb{R}}\{\mathcal{W}(N, r, t)-u r\}
$$

Notice that the variational formulation of GSOM models (3.14) is only available if and only if $r \mapsto \mathcal{W}(N, r, t)$ is concave such that the Legendre-Fenchel transform of $\mathcal{M}$ gives back the function $\mathcal{W}$.

In the previous formulation (3.14), $\mathcal{J}$ is the locus of initial and boundary conditions and $\xi\left(N_{0}, t_{0}\right)$ is the initial data of the position of vehicle $N_{0}$ at time $t_{0}$ for all $\left(N_{0}, t_{0}\right) \in \mathcal{J}$.

The advantage of the variational formulation (3.14) is that it expresses the position of the vehicle indexed $N$ at time $T$ as an optimal control problem which only depends on the initial position at time $t_{0}$. To find the optimal trajectories of such a problem, it suffices to use the minimum principle of Pontryagin. Moreover, notice that the optimal trajectories are given by the characteristics (Eulerian and Lagrangian characteristics are equal). We have:

$$
\left\{\begin{array}{l}
\dot{N}=\partial_{r} \mathcal{W}(N, r, t) \\
\dot{r}=-\partial_{N} \mathcal{W}(N, r, t)
\end{array}\right.
$$




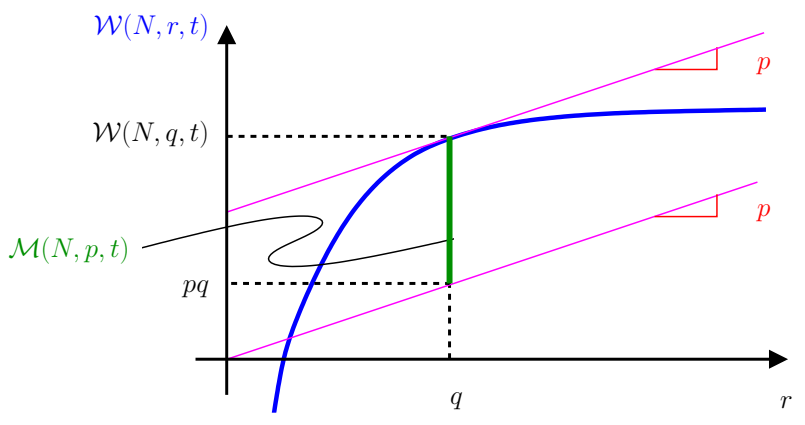

Figure 4: Legendre transform $\mathcal{M}$ of function $\mathcal{W}$.

where $u=\dot{N}$ is the command of the optimal control problem (3.14). The system (3.16) is a simple system of ODEs in the $(N, r)$ plane.

Notice that in what follows, we will restrict ourselves to the variational formulation of GSOM models in Lagrangian setting. Indeed as the driver attribute $I$ is propagated at the traffic speed, the Lagrangian point of view seems the most well-suited framework to deal with the specific dynamics of $I$. Moreover it is easier to deal with trajectories data given by mobile sensing devices.

\subsection{Review of computational methods for GSOM models}

There already exists some works on computational methods for models of the GSOM family [41, 50. However these existing methods are developed in the Eulerian framework which does not seem to be the best one to deal with Lagrangian data. Moreover the paper [50] deals with the LWR model with bounded acceleration which is a very specific GSOM model. The algorithm is very similar to the ones developed in [7, 45] but it is not applicable to general models of the GSOM family.

In [41] the authors underline that the variational formulation has the advantage of developing efficient numerical schemes that provide solutions for problems which incorporate moving elements in traffic flow models such as moving bottlenecks or probe vehicles. This remark shows also the interest of the Lagrangian setting (compared to the Eulerian viewpoint) which moves within the traffic stream. However their numerical scheme is based on a discretization of Eulerian time and space domain. The idea of the scheme (close to dynamic programming) is then to update the cumulative quantities

$$
N_{\rho}:=\int_{x}^{+\infty} \rho(y, t) d y \quad \text { and } \quad N_{s}:=\int_{x}^{+\infty} s(y, t) d y,
$$

on the vertexes of a mesh (whatever its shape if this mesh is dense enough) following the optimal paths in an iterative way because of the coupling of the equations to solve. The values of $\rho$ (resp. $s$ ) are deduced from a first order approximation of the derivative of $N_{\rho}$ (resp. $N_{s}$ ). Notice that this computational method is exact only for special cases.

Up to our best knowledge, there is no existing work on computational methods for models of the GSOM family in the Lagrangian framework.

\section{Computational aspects}

We are now interested in numerical methods to efficiently recover the solution of the HamiltonJacobi problem (3.13). The computation of numerical solutions of the HJ equation has already attracted an important interest in the mathematical community. The majority of numerical 
schemes which were proposed to solve HJ equations are based on finite difference methods, Semi-Lagrangian schemes and discontinuous Galerkin methods (the interested reader is referred to [7, 11] and references therein).

\subsection{A finite difference scheme}

We first describe a numerical scheme based on particle and time discretization which was originally presented in [34]. It introduces a uniform grid of the $t-n$ domain based on fixed time and label steps $\Delta t$ and $\Delta n$. We define for any $n, t \geq 0$

$$
\mathcal{X}_{n}^{t}:=\mathcal{X}(n \Delta n, t \delta t) .
$$

As usual with finite difference scheme, we need to introduce a Courant-Friedrichs-Lewy (CFL) condition to ensure the scheme to be monotone and convergent

$$
\frac{\Delta n}{\Delta t} \geq \max _{r, N, t} \mathcal{W}(N, r, t) .
$$

Then we consider the classical first order finite difference scheme as follows

$$
\mathcal{X}_{n}^{t+1}=\mathcal{X}_{n}^{t}+\Delta t \mathcal{W}\left(n \Delta n, \frac{\mathcal{X}_{n+1}^{t}-\mathcal{X}_{n}^{t}}{\Delta n}, t \Delta t\right) .
$$

By construction the above scheme can be interpreted as the seminal Godunov (finite volume) scheme (4.18) for the Lagrangian formulation of the GSOM model (see [11])

$$
r_{n}^{t+1}=r_{n}^{t}+\frac{\Delta t}{\Delta n}\left[\mathcal{W}_{n}^{t}-\mathcal{W}_{n+1}^{t}\right],
$$

where we have defined the discrete spacing and respectively the numerical speed as follows

$$
\left\{\begin{array}{l}
r_{n}^{t}:=\frac{\mathcal{X}_{n+1}^{t}-\mathcal{X}_{n}^{t}}{\Delta n}, \\
\mathcal{W}_{n}^{t}:=\mathcal{W}\left(n \Delta n, r_{n}^{t}, t \Delta t\right) .
\end{array}\right.
$$

The upstream and downstream boundary conditions for the finite difference scheme (4.17) are fully described in [34]. They match the Bardos-LeRoux-Nedelec or Dubois-LeFloch boundary conditions and it was already shown that such conditions are equivalent to prescribe supply and demand conditions (see [34] and references therein).

The method of finite differences does not enjoy the semi-analytical expression of the solution of the HJ equation (3.13) thanks to Lax-Hopf formula (4.19). We then provide a semi-analytical algorithm to compute the solution based on that representation formula.

\subsection{A "grid free" scheme}

The idea to compute the solution of the Eulerian GSOM model (3.10) is to recast it in its Lagrangian form (3.11). As it was shown in the previous section, the position $\mathcal{X}$ of vehicle $N$ at time $t$ solves the Hamilton-Jacobi equation (3.13). As explained in [34, the Initial and Boundary Value Problem (3.13) admits a quite simple representation formula (3.14), very similar to the Hopf-Lax formula presented for the LWR model.

The Hopf-Lax formula (3.14) can be simplified because as it is well-known in optimal control theory, the optimal trajectories for which the minimum is attained are the characteristics 
(3.16). Hence we have to solve the following system of coupled ODEs (3.16). Then the generalized Hopf-Lax formulation (3.14) can be recast as follows

$$
\begin{aligned}
\mathcal{X}\left(N_{T}, T\right)= & \min _{\left(N_{0}, r_{0}, t_{0}\right)} \int_{t_{0}}^{T} \mathcal{M}\left(N, \partial_{r} \mathcal{W}(N, r, t), t\right) d t+\xi\left(N_{0}, t_{0}\right), \\
& \begin{array}{l}
\dot{N}(t)=\partial_{r} \mathcal{W}(N, r, t) \\
\dot{r}(t)=-\partial_{N} \mathcal{W}(N, r, t) \\
N\left(t_{0}\right)=N_{0}, \quad r\left(t_{0}\right)=r_{0}, \quad N(T)=N_{T} \\
\left(N_{0}, r_{0}, t_{0}\right) \in \mathcal{K}
\end{array}
\end{aligned}
$$

where $\mathcal{K}$ is the set of initial/boundary values obtained by combining the initial values of $\mathcal{J}$ with the initial/boundary value $r_{0}$ deduced from the initial values $\xi\left(N_{0}, t_{0}\right)$.

According to the principle of inf-morphism property [2, 3, 6, 7, if the initial/boundary condition data $\xi$ are located on a union (non necessarily disjoint) of sets

$$
\mathcal{K}=\bigcup_{l} \mathcal{K}_{l}
$$

it suffices to solve partial problems on each set $\mathcal{K}_{l}$ and to compute the minimum of the solutions of these sub-problems

$$
\mathcal{X}\left(N_{T}, T\right)=\min _{l} \mathcal{X}_{l}\left(N_{T}, T\right),
$$

with

$$
\begin{aligned}
\mathcal{X}_{l}\left(N_{T}, T\right):= & \min _{\left(N_{0}, r_{0}, t_{0}\right)} \int_{t_{0}}^{T} \mathcal{M}\left(N, \partial_{r} \mathcal{W}(N, r, t), t\right) d t+\xi\left(N_{0}, t_{0}\right) \\
& \mid \begin{array}{l}
\dot{N}_{l}(t)=\partial_{r} \mathcal{W}\left(N_{l}, r_{l}, t\right) \\
\dot{r}_{l}(t)=-\partial_{N} \mathcal{W}\left(N_{l}, r_{l}, t\right) \\
N_{l}\left(t_{0}\right)=N_{0}, \quad r_{l}\left(t_{0}\right)=r_{0}, \quad N_{l}(T)=N_{T} \\
\left(N_{0}, r_{0}, t_{0}\right) \in \mathcal{K}_{l}
\end{array}
\end{aligned}
$$

In the remaining of this article, we will apply the inf-morphism property by considering initial and boundary conditions which are piecewise affine (PWA). Thus we will calculate the solution generated by each piece using (4.21) and then apply (4.20) in order to obtain the solution of (3.14).

\subsection{Algorithm for piecewise affine value conditions}

Hereafter, we study separately the different elements which contribute to the value of the solution of (3.13) in its Lagrangian setting. We distinguish

- the initial condition at time $t=t_{0}$,

- the "upstream" boundary condition that is the trajectory of the first vehicle $N=N_{0}$ traveling on the section,

- and internal boundary conditions given by cumulative vehicles counts at fixed location $\mathcal{X}=x_{0}$.

While the Lax-Hopf algorithm can handle infinite horizon problems either in the Eulerian or in the Lagrangian framework, we restrict ourselves to finite values for a convenient numerical implementation. We then assume that $N \in\left[N_{0}, N_{\max }\right]$ with $N_{\max }<+\infty$ and similarly $t \in\left[t_{0}, t_{\max }\right]$ with $t_{\max }<+\infty$. This restriction to a finite horizon implies to not consider the whole domain of influence generated by the initial and boundaries conditions. 


\subsubsection{Initial conditions}

In this case, at $t=t_{0}$, the positions $\xi\left(n, t_{0}\right)$ of vehicles $n$ are given. We have that

$$
r_{0}(N)=-\partial_{N} \xi\left(N, t_{0}\right), \quad \text { for any } N .
$$

The initial conditions for the characteristics are the couples $\left(N, r_{0}(N)\right)$.

First, we need to discretize the set of vehicles labels into intervals $\left[n_{p}, n_{p+1}\right]$ of length $\Delta n$, for $p=1, \ldots, P$, in such a way that the dynamics $\varphi$ of the driver attribute $I$ can be approximated by $\varphi_{p}$ in the interval $\left[n_{p}, n_{p+1}\right]$

$$
\varphi(N, I, t)=\varphi_{p}(I, t), \quad \text { for any } \quad N \in\left[n_{p}, n_{p+1}\right] .
$$

If the discrete step $\Delta n$ is small enough, we could also assume that for any $N \in\left[n_{p}, n_{p+1}\right]$ the initial data are piecewise constant

$$
\left\{\begin{array}{l}
I\left(N, t_{0}\right)=I_{0, p} \\
r\left(N, t_{0}\right)=r_{0, p} .
\end{array}\right.
$$

We will now first define properly the initial condition and then we calculate the solution generated by the $p^{\text {th }}$ component $(p=1, \ldots, P)$.

Definition 4.1 (PWA initial condition)

Let $t_{0} \geq 0$ be fixed. Then the $p^{\text {th }}$ component of the initial condition $(p=1, \ldots, P)$ is given by

$$
\mathcal{X}^{\text {ini }}\left(N, t_{0}\right)=r_{0, p} N+\alpha_{p}, \quad \text { for any } \quad N \in\left[n_{p}, n_{p+1}\right] .
$$

To ensure continuity of the initial data $\mathcal{X}^{\text {ini }}$ on $\left[N_{0}, N_{\text {max }}\right]$, we require that

$$
\alpha_{p}=-r_{0, p} n_{p}+\sum_{l=1}^{p-1} r_{0, l}\left(n_{l+1}-n_{l}\right), \quad \text { for any } \quad p=1, \ldots, P .
$$

To considerably simplify the presentation, we will restrict ourselves to the case of systems with no relaxation that is the dynamics $\varphi_{p}$ of driver attributes is trivial on any interval $\left[n_{p}, n_{p+1}\right]$ with $p=1, \ldots, P$. In a first step, we want to compute the characteristics generated by the initial conditions $\left(I\left(N, t_{0}\right)=i_{0, p}\right.$ and $r\left(N, t_{0}\right)=r_{0, p}$ given on the interval $\left[n_{p}, n_{p+1}\right]$ at time $\left.t_{0}\right)$. In the general case, we would have

$$
I(N, t)=i_{p}(t) \quad \text { for any } \quad N \in\left[n_{p}, n_{p+1}\right] .
$$

In the following, we consider a characteristic denoted by $N(t)$ for $t \in\left[t_{0}, t_{\max }\right]$ and we distinguish two cases according to the location of this characteristic.

On the interval $\mathbf{N} \in] \mathbf{n}_{\mathbf{p}}, \mathbf{n}_{\mathbf{p}+\mathbf{1}}[$.

Consider $\Omega_{p}:=\{t \mid N(t) \in] n_{p}, n_{p+1}[\}$ for any $p=1, \ldots, P$. We then have to solve the following nonlinear first order ODE

$$
\left\{\begin{array}{l}
\dot{I}_{p}(t)=\varphi_{p}\left(I_{p}(t)\right), \quad \text { for } \quad t \in \Omega_{p} \\
I_{p}\left(t_{0}\right)=I_{0, p}
\end{array}\right.
$$

If we assume that the dynamics is null, then it obviously follows that $\dot{I}_{p}=0$ which leads to

$$
I_{p}(t)=I_{0, p} \quad \text { for any } \quad t \in \Omega_{p} .
$$

In traffic modeling, this choice is relevant if the driver attribute $I$ represents for example some origin-destination (OD) information or if it characterizes vehicles kind. The driver attribute is conserved at any time and along any characteristic inside each strip $\left[n_{p}, n_{p+1}\right]$ of length $\Delta n$. 
On the interval $] n_{p}, n_{p+1}\left[\right.$, we have that $I(N, t)=i_{p}(t)$ is independent of $N$. Then from (3.16), we deduce

$$
\begin{aligned}
\dot{r} & =-\partial_{N} \mathcal{W}(N, r, t) \\
& =-\partial_{I} \mathcal{V}(N, I(N, t)) \partial_{N} I(N, t) \\
& =0 .
\end{aligned}
$$

Thus $r(N, t)=r_{0, p}$ for any $t \in \Omega_{p}$. Hence it simply suffices to solve the other ODE in (3.16) that is

$$
\dot{N}=\partial_{r} \mathcal{W}(N, r, t)
$$

to get the equation of the characteristic on the interval

$$
N(t)-N(u)=\int_{u}^{t} \partial_{r} \mathcal{W}(N, r, \tau) d \tau \quad \text { for any } \quad t, u \in \Omega_{p} .
$$

At the edge $\mathbf{N}=\mathbf{n}_{\mathrm{p}}$.

The edge $N=n_{p}$ separates two different traffic states that rely on two different speed-spacing fundamental diagrams. We need to take care of the kinematic waves that can appear at this locus. As illustrated on Figure 5, we set the characteristic speed related to the spacing $r_{0, p}$ as follows

$$
\nu_{p}:=\partial_{r} \mathcal{V}\left(r_{0, p}, I_{0, p}\right),
$$

and we also define the speed of the refracted" characteristic wave through the discontinuity of $I$ such that

$$
\nu_{p}^{*}:=\partial_{r} \mathcal{V}\left(r_{0, p}^{*}, I_{0, p}\right) .
$$

This characteristic speed is related to a new spacing value

$$
r_{0, p}^{*}= \begin{cases}\mathcal{V}^{-1}\left(\mathcal{V}\left(r_{0, p-1}, I_{0, p-1}\right), I_{0, p}\right) & \text { if } \mathcal{V}\left(r_{0, p-1}, I_{0, p-1}\right)<\max _{r} \mathcal{V}\left(r, I_{0, p}\right), \\ +\infty & \text { else. }\end{cases}
$$

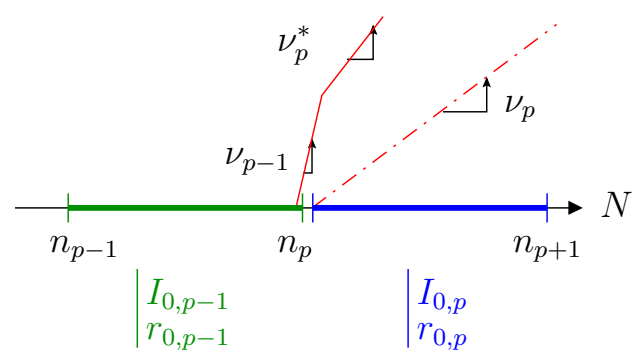

Figure 5: Schematic view of what happens for the characteristic wave generating from the edge $N=n_{p}$ and passing through a discontinuity of $I$.

Regarding the values of the initial spacing $r_{0, p}$ and $r_{0, p-1}$, we can distinguish two cases that could occur starting to an edge $N=n_{p}$ :

- either $\nu_{p}^{*}>\nu_{p}$ and in this case there will be a shock wave. The characteristics carrying respectively the initial states $r_{0, p-1}$ and $r_{0, p}$ will cross each other, offering a partial superposition of both characteristics domains (see (a) on Figure 6). The solution is then simply computed thanks to the inf-morphism property. Hence we only consider the two characteristics (indicated as (1) and (2) on Figures 8, 9 and 11) that encompass the domain of influence generated by the initial spacing $r_{0, p}$. 
- or $\nu_{p}^{*}<\nu_{p}$ and in this case there will be a rarefaction fan. The characteristics waves carrying the initial states $r_{0, p-1}$ and $r_{0, p}$ diverge (see (b) on Figure 6). The solution on the area between both extreme characteristics may not be computed and even the infmorphism property will not be able to recover it. That is why we need to consider the first two characteristics (as above) and a third wave corresponding to the first characteristic matching with the initial state $r_{0, p-1}$ (labeled (1) on Figures 8, 9 and 11).

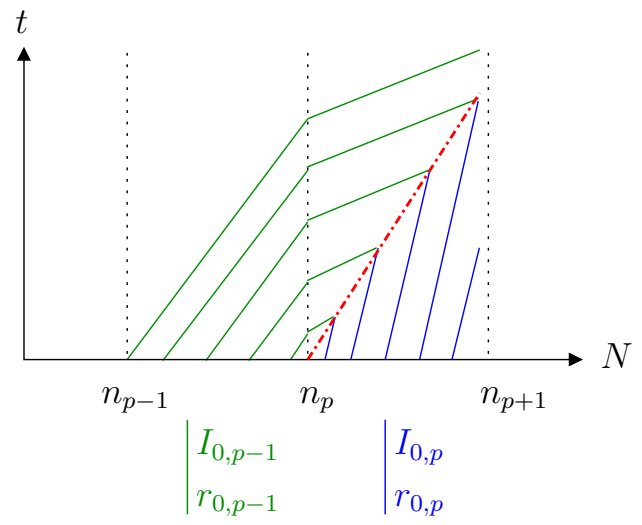

(a) Case of a shock wave

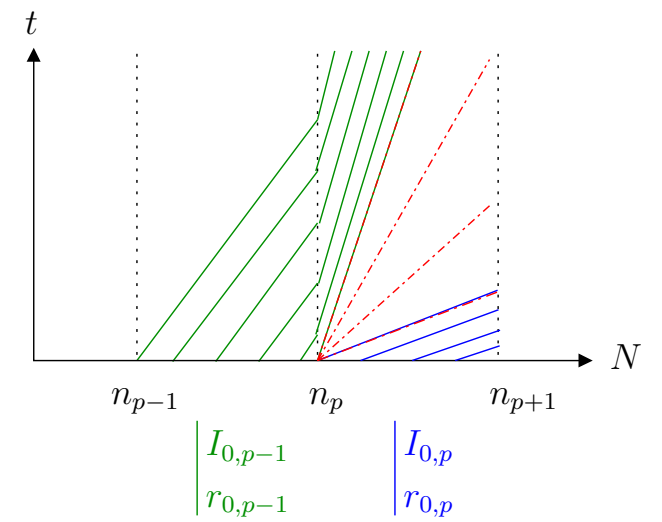

(b) Case of a rarefaction fan

Figure 6: Characteristics through a discontinuity of $I$ of $r$.

Notice that by convention we add the rarefaction wave (1) to the solution generated by the initial condition on $\left[n_{p}, n_{p+1}\right]$ for any $p=1, \ldots, P$.

\section{Remark 4.2 (Degenerated case for characteristics through a discontinuity of $I$ )}

When passing through a discontinuity of I (assume $(a)$ and $(b)$ the states on both sides of the discontinuity as illustrated on Figure [7), the characteristics speed may be changed. Nevertheless it is well known (Rankine-Hugoniot jump condition) that through such a discontinuity, the traffic speed should be unmodified

$$
v^{(a)}:=\mathcal{V}\left(r^{(a)}, I^{(a)}\right)=\mathcal{V}\left(r^{(b)}, I^{(b)}\right)=: v^{(b)} .
$$

In case of $v^{(a)}>v_{\max }^{(b)}:=\max _{r} \mathcal{V}\left(r, I^{(b)}\right)$, it is obvious that the equality between these two speeds cannot be complied. In an Eulerian setting, it means that the group of upstream vehicles (b) cannot accelerate enough (even increasing their speeds to $v_{\max }^{(b)}$ ) to catch up with the downstream vehicles (a). This induces the apparition of a vacuum area between both groups of vehicles. There is a rarefaction wave as vehicles $(b)$ accelerate to attain their top speed and a shock wave following the last vehicle of type $(a)$. Notice that the states $\left(a_{\infty}\right)$ and $\left(b_{\infty}\right)$ defined on Figure 7) coincide for $r=+\infty$ (or equivalently at $\rho=0$ ).

The contributions of the initial condition defined on $\left[n_{p}, n_{p+1}\right] \times\left\{t_{0}\right\}$ for any $1 \leq p \leq P$ are then computed as follows:

(i) Initialize the values of the solution $\mathcal{X}$ at $+\infty$ on the whole computational domain.

(ii) Determine the number of characteristics to compute (two or three) according to what could occur at the edge $N=n_{p}$.

(iii) Compute the equation $N(t)$ of each characteristic while $t \leq t_{\max }$ and $N \leq N_{\max }$.

(iv) Calculate the (exact) solution $\mathcal{X}_{p}$ all along each computed characteristic generated by the interval $\left[n_{p}, n_{p+1}\right] \times\left\{t_{0}\right\}$, namely characteristics (1) and (2) and (1) whenever it appears 


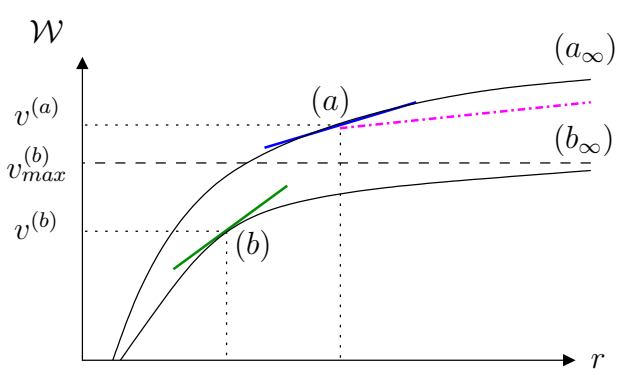

(a) Speed-spacing fundamental diagram

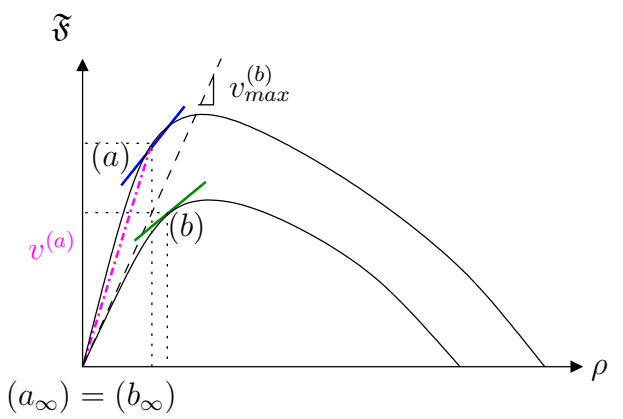

(a) Flow-density fundamental diagram

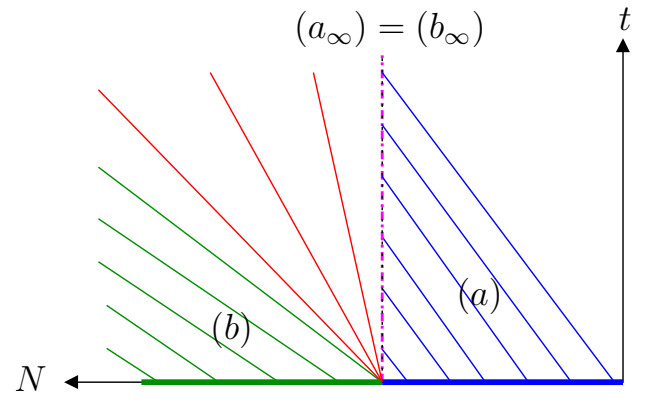

(b) Some characteristics in Lagrangian viewpoint

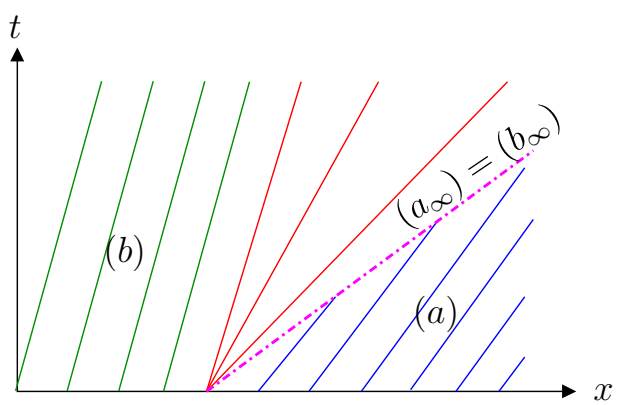

(b) Some characteristics in Eulerian viewpoint

Figure 7: Critical "vacuum" case appearing from special condition values.

(see Figure 8). In this aim we use the generalized Lax-Hopf formula (4.21) which gives that

$$
\dot{\mathcal{X}}(t)=\mathcal{M}(N(t), \dot{N}(t), t) .
$$

The interested reader is referred to [5, 10, 43] for additional information about the Legendre-Fenchel transform and fast algorithms for its numerical computation.

(v) Compute the exact value at any point within the characteristics fan (delimited by characteristics (1) and (2) on Figure 8) using the fact that for any point $(N, t)$ belonging to the characteristic fan, the position at this point can be deduced by a simple translation of the position on characteristic (1) (see Figure 8). Indeed we have for any $N \in\left[n_{p}, n_{p+1}\right]$ and any $t \in \Omega_{p}$

$$
\begin{aligned}
\mathcal{X}(N, t) & =\mathcal{X}\left(N, t_{N}^{(1)}\right)+\int_{t_{N}^{(1)}}^{t} \mathcal{V}(r(N, \tau), I(N, \tau)) d \tau \\
& =\mathcal{X}\left(N, t_{N}^{(1)}\right)+\mathcal{V}\left(r_{0, p}, I_{0, p}\right)\left(t-t_{N}^{(1)}\right),
\end{aligned}
$$

where we recall that $\varphi_{p}(I)=0$. The time $t_{N}^{(1)}$ corresponds to the time when the characteristic (1) crosses the line $N$.

(vi) In the case of a rarefaction fan, evaluate the value of $\mathcal{X}$ at each point within the influence domain of the considered initial condition (illustrated on Figure 8) by an interpolation technique based on triangular meshes (the value at each triangle vertex is exact).

\subsection{2 "Upstream" boundary conditions}

In the Lagrangian setting, boundary conditions describe floating vehicles conditions in Eulerian setting. Indeed such a condition is equivalent to consider the trajectory $t \mapsto \xi\left(N_{0}, t\right)$ of a given 


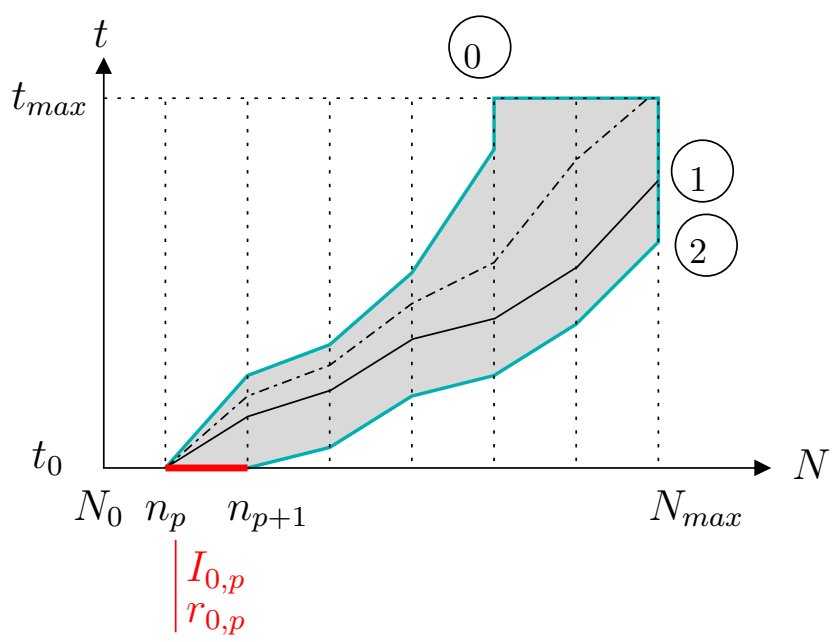

Figure 8: Domain of influence of the initial data $\left[n_{p}, n_{p+1}\right] \times\left\{t_{0}\right\}$.

vehicle $N_{0}$. Then we have that

$$
\mu_{0}(t):=\partial_{t} \xi\left(N_{0}, t\right)=\mathcal{V}\left(r_{0}\left(N_{0}, t\right), I\left(N_{0}, t\right)\right), \quad \text { for any } t \geq t_{0},
$$

which can be solved and yields a unique solution (because $\mathcal{V}$ is strictly increasing)

$$
r_{0}(t)=\mathcal{V}^{-1}\left(\mu_{0}(t), I\left(N_{0}, t\right)\right), \quad \text { for any } t \geq t_{0} .
$$

The initial conditions for the characteristics are the couples $\left(N_{0}, r_{0}(t)\right)$.

As for initial condition, we introduce a discrete time step $\Delta t$ and we consider a full discretization of the time domain $\left[t_{0}, t_{\max }\right]$ into segments $\left[t_{q}, t_{q+1}\right]$ with $q=1, \ldots, Q$.

\section{Definition 4.3 (PWA upstream boundary condition)}

Let $N_{0} \geq 0$ be fixed. Then the $q^{\text {th }}$ component of the upstream boundary condition $(q=1, \ldots, Q)$ is given by

$$
\mathcal{X}^{u p}\left(N_{0}, t\right)=v_{0, q} t+\beta_{q}, \quad \text { for any } t \in\left[t_{q}, t_{q+1}\right] .
$$

To ensure continuity of the upstream boundary data $\mathcal{X}^{\text {ini }}$ on $\left[t_{0}, t_{\text {max }}\right]$, we require that

$$
\beta_{q}=-v_{0, q} t_{q}+\sum_{l=1}^{q-1} v_{0, l}\left(t_{l+1}-t_{l}\right), \quad \text { for any } \quad q=1, \ldots, Q .
$$

The contributions of upstream boundary conditions are computed in a similar way than those of initial condition. The domain of influence of upstream boundary conditions seems to be simply the reverse of the one of initial condition (see Figure 9).

Remark 4.4 (Extension to any individual trajectory condition) Any Lagrangian data giving the trajectory of a vehicle $N^{*}$ with $N^{*} \in\left[N_{0}, N_{\text {max }}\right]$ will strictly generate the same type of calculations than the ones described for the upstream boundary condition. It is then easy to deal with data coming from mobile sensors moving within the traffic stream.

\subsubsection{Internal boundary conditions}

Internal boundary condition is here understood in the Lagrangian framework. It does not correspond to internal vehicle trajectories which can also be incorporated in the algorithm (see the previous subsection). Notice that internal boundary condition is called mixed condition 


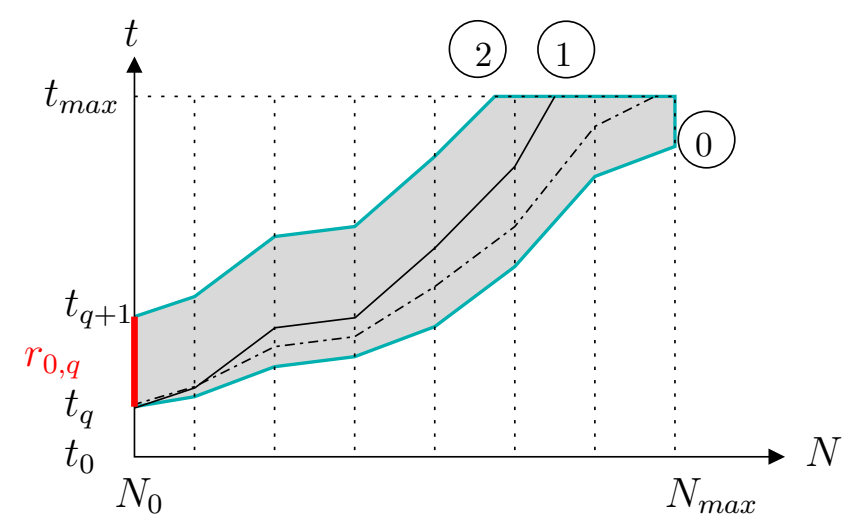

Figure 9: Domain of influence of the upstream boundary data $\left\{N=N_{0}\right\} \times\left[t_{q}, t_{q+1}\right]$.

in [34] to avoid any misunderstanding.

We assume that the data comes from vehicles $N(t)$ located at a point $\xi(N(t), t)$ at time $t$. Thus we get that

$$
\frac{d}{d t} \xi(N(t), t)=-\dot{N}(t) r_{0}(t)+\mathcal{W}\left(N(t), r_{0}(t), t\right)
$$

As not all data are compatible, we need to introduce compatibility conditions. As we have

$$
\max _{r}\{-\dot{N}(t) r+\mathcal{W}(N(t), r, t)\}=\mathcal{M}(N(t), \dot{N}(t), t),
$$

hence the equation yielding $r_{0}(t)$ admits a solution only if

$$
\frac{d}{d t} \xi(N(t), t) \leq \mathcal{M}(N(t), \dot{N}(t), t)
$$

Let us describe the specific situation of $\dot{\xi}(t)=0$ and $\dot{N}(t) \geq 0$ for any time $t$. This case occurs if the data originates at a fixed measurement point $x_{0}$ like a fixed detector data that is $\xi(N(t), t)=x_{0}$ for all time $t$. Then $N(t)$ represents the cumulative flow at point $x_{0}$. In this case the above compatibility condition is satisfied, there are two solutions, one under-critical and the other over-critical. Recall that the speed-spacing fundamental diagram $\mathcal{W}$ depends on the vehicle $N$.

If we propagate both pairs of characteristics, then the inf-morphism property will automatically select a single solution which matches the over-critical situation (because the speed is lower than in the under-critical situation). It may happen that the congested solution is not the good one. Then to avoid any mistake, we assume that the fixed detector gives the cumulative flow and also the traffic flow speed. In this way, we can select one traffic state only.

In our case, we are interested in including some Eulerian data coming from classical fixed sensors like inductive loop detectors. In this aim, Eulerian data become internal boundary condition into the Lagrangian framework. We assume that a Eulerian sensor located at a fixed position $x_{0}$ gives us the incremental cumulative vehicles count which is then interpolated in a piecewise affine function $N(t)$ for $t \in\left[t_{0}, t_{\max }\right]$.

\section{Definition 4.5 (PWA internal boundary condition)}

Let $x_{0} \geq 0$ be fixed. Then the $p^{\text {th }}$ component of the internal boundary condition $(p=1, \ldots, P)$ is given by

$$
\mathcal{X}^{\text {int }}(n, t)=x_{0}, \quad \text { for } \quad n=\mathfrak{f}_{0, p} t+\gamma_{p} \quad \text { and } \quad t \in\left[\hat{t}_{p}, \hat{t}_{p+1}\right] \text {, }
$$




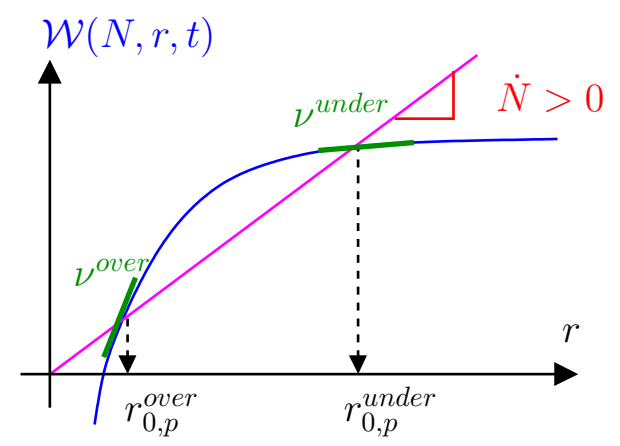

(a) Speed-spacing fundamental diagram

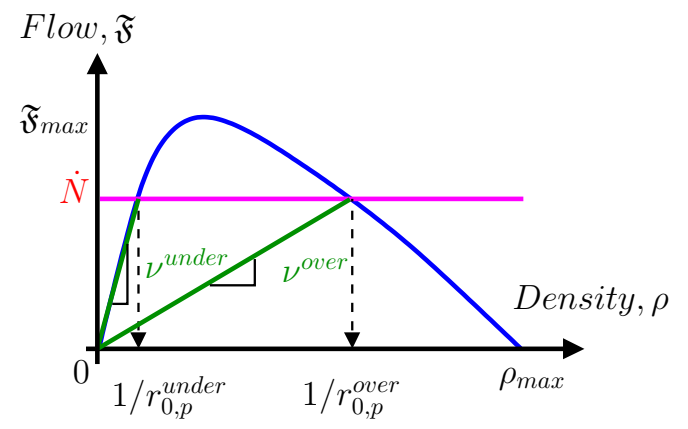

(b) Flow-density fundamental diagram

Figure 10: Existence of two solutions corresponding to a condition $\dot{\xi}(t)=0$ and $\dot{N}(t) \geq 0$.

where we define $\hat{t}_{p}$ for any $p$ as the time such that $n=n_{p}$. To ensure continuity of the trajectory $t \mapsto n(t)$ on which the internal boundary data $\mathcal{X}^{\text {int }}$ is prescribed, we require that

$$
\gamma_{p}=-\mathfrak{f}_{0, p} \hat{t}_{p}+\sum_{l=1}^{p-1} \mathfrak{f}_{0, l}\left(\hat{t}_{l+1}-\hat{t}_{l}\right), \quad \text { for any } \quad p=1, \ldots, P .
$$

We assume that $N$ is piecewise affine on each discrete segments $\left[n_{p}, n_{p+1}\right]$ that is equivalent to say that $\dot{N}$ is piecewise constant (i.e. constant in each strip $\left[n_{p}, n_{p+1}\right]$ for $p=1, \ldots, P$ ). It is easy to deal with this case for the algorithm because the computational steps are similar to both previous cases for initial and upstream boundary conditions.

The only difference resides in the fact that we have a characteristic fan that matches either the under-critical or the over-critical traffic state. It is simple to verify that in the first (undercritical) case, the $p^{\text {th }}$ component generates characteristics which are emitted with speeds $\nu^{\text {under }}>\dot{N}$ while it is the inverse in the other case (see Figure 11). From a traffic point of view it is relevant with observations stating that a congestion wave will spread backward at a slower speed than a decompression wave.

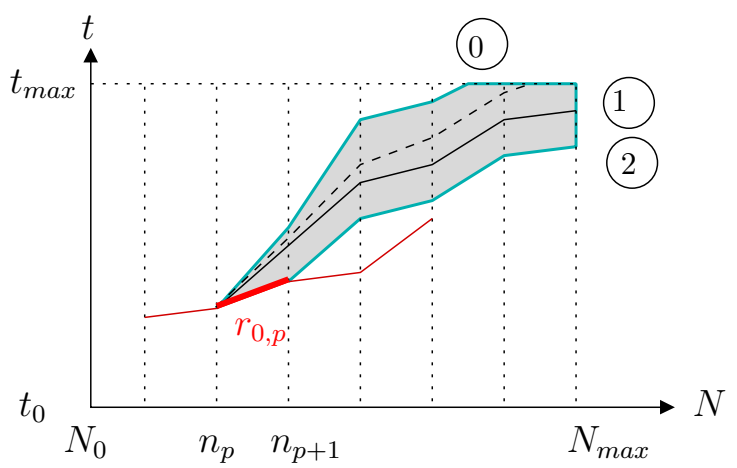

(a) Generation by the over-critical value

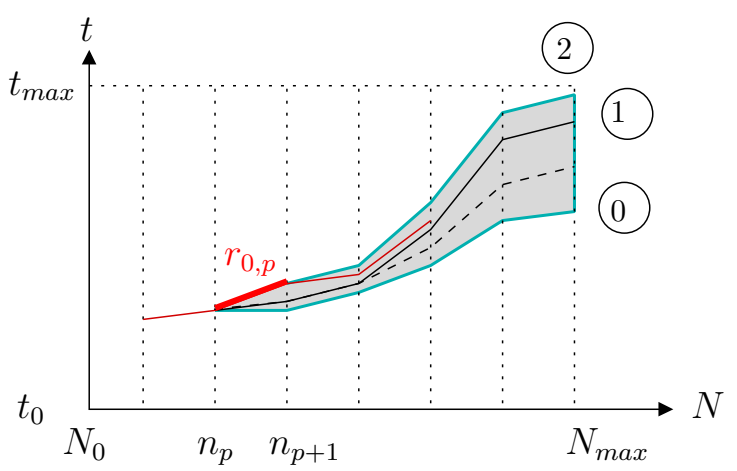

(b) Generation by the under-critical value

Figure 11: Domain of influence of the internal boundary data.

\subsection{Recapitulation of the algorithm}

The different elements of the computations for piecewise affine value conditions are recapitulated in the following pseudo-code (see Algorithm 1). 


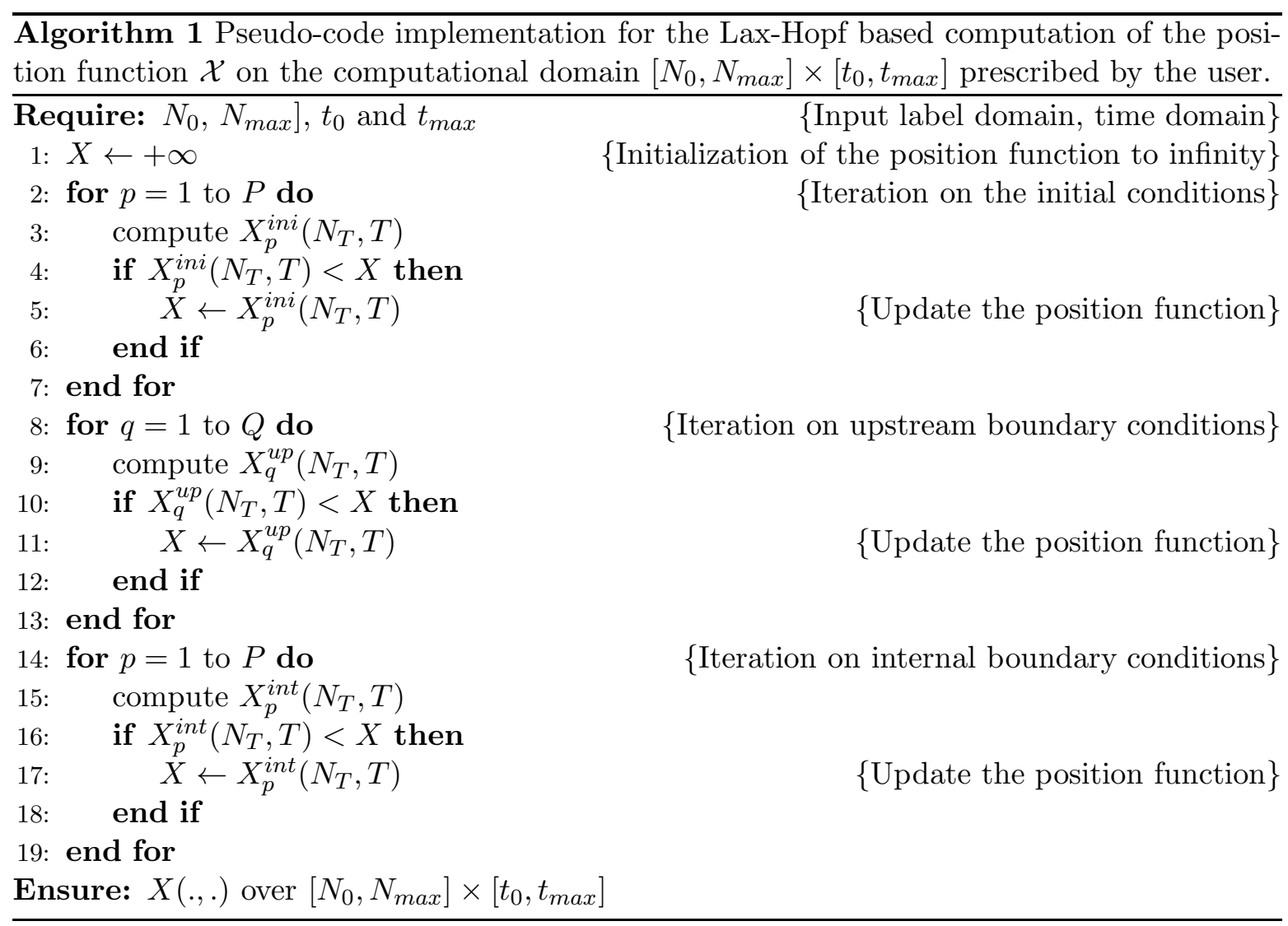

Remark 4.6 This pseudo-code underlines the property of the Lax-Hopf algorithm to treat in a parallel way the different value conditions (initial, upstream boundary and internal boundary).

\section{$5 \quad$ Numerical example}

In order to simplify the computations and to ease the presentation of the following example, we consider that the driver attribute $I$ is piecewise constant with respect to $N$ at initial time $t=t_{0}$. It means that there exist some platoons of vehicles which share the same driver attribute. It could be for example some vehicles of the same kind (cars and trucks), or vehicles that go to the same destination, or vehicles that have the same desired maximal speeds.

We consider for this numerical example three classes of drivers according to the value taken by the driver attribute $I$, from 1 to 3 . The corresponding fundamental diagrams are given on Figure 12. The driver attribute $I=1$ can match with trucks as the maximal speed $25 \mathrm{~m} \cdot \mathrm{s}^{-1}$ is lower than in the two other cases and the sensitivity to variations of spacing is less important as well. The driver attributes $I=2$ and 3 can represent cars with different desired maximal speeds (resp. $35 \mathrm{~m} . \mathrm{s}^{-1}$ and $30 \mathrm{~m} . \mathrm{s}^{-1}$ ) and different characteristics of acceleration, due to sensitivity to variations of spacing.

We consider piecewise affine initial conditions that is we prescribe each vehicle position $X(t, n)$ at time $t=t_{0}$ for $n \in\left[N_{0}, N_{\max }\right]$. The initial condition for this numerical test are plotted on Figure 13.

Moreover we prescribe piecewise affine upstream boundary condition that is the trajectory $X(t, n)$ of the first vehicle $N=N_{0}$ for $t \in\left[t_{0}, t_{\max }\right]$. This upstream boundary condition is illustrated on Figure 14,

The solution is thus given by applying the computation algorithm described in Algorithm 1. The simulations deal with 30 vehicles during 5 minutes. The numerical results are plotted on Figure 15, 

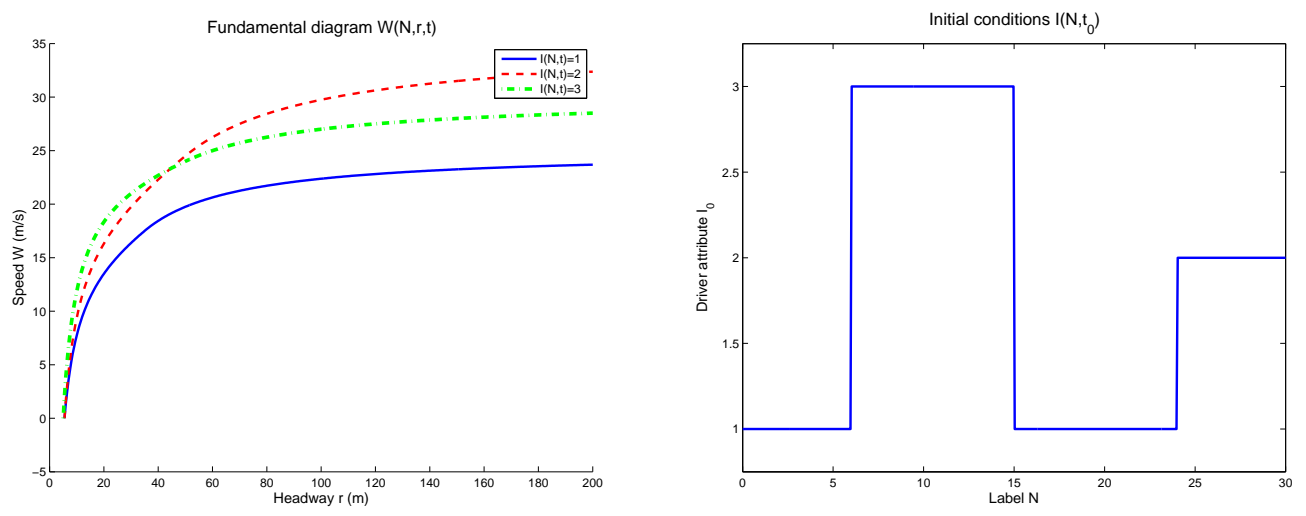

Figure 12: Speed-spacing fundamental diagram $\mathcal{V}(r, I)$ (left) and initial values of driver attribute $I\left(N, t_{0}\right)$ (right).

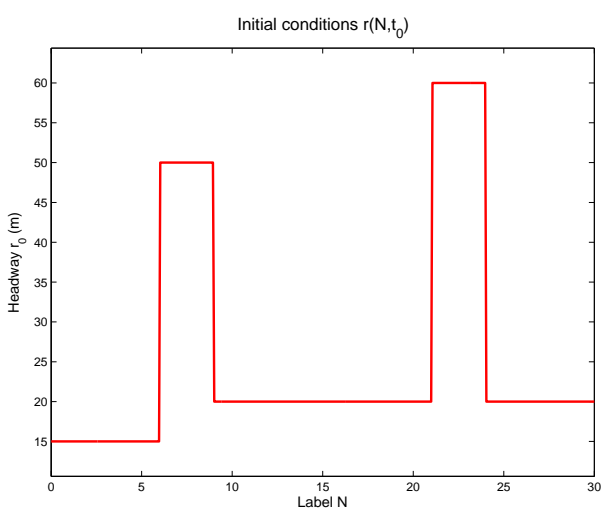

(a) Initial headways $r\left(N, t_{0}\right)$

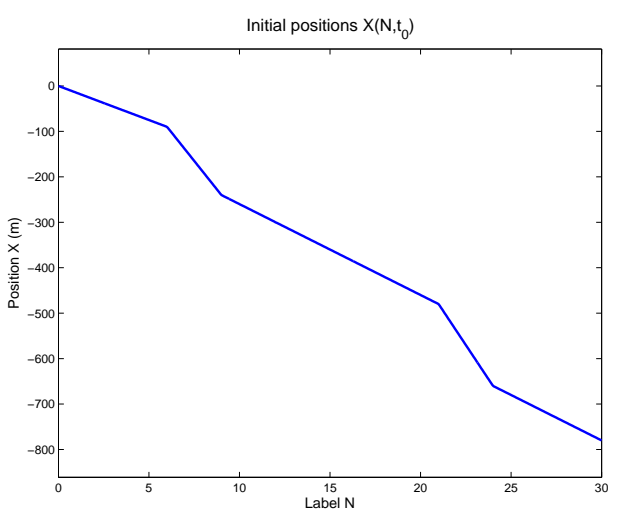

(b) Initial positions of vehicles $\mathcal{X}\left(N, t_{0}\right)$

Figure 13: Initial conditions for the GSOM PDE at $t=t_{0}$.

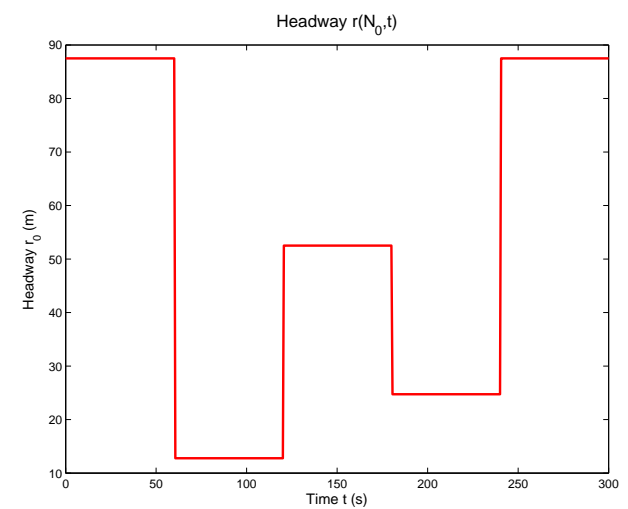

(a) Boundary headway $r\left(N_{0}, t\right)$

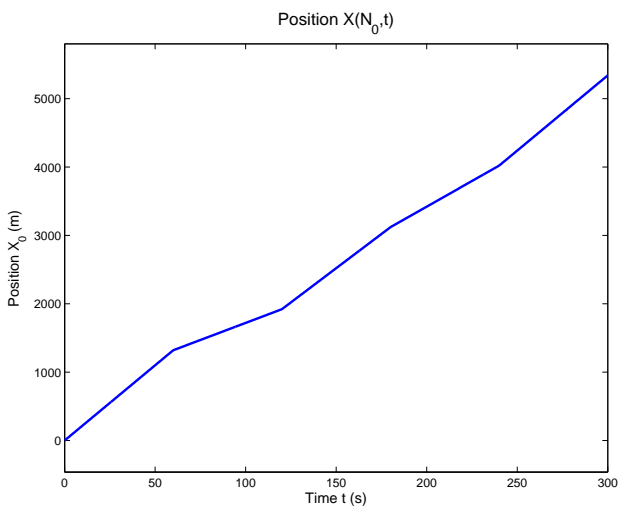

(b) First vehicle trajectory $\mathcal{X}\left(N_{0}, t\right)$

Figure 14: Boundary conditions for the GSOM PDE at $N=N_{0}$.

In the numerical example above, we do not consider real data assimilation. The next step for numerical results should be the assimilation of real data in a model of the GSOM family. As it was described in the previous section, vehicles trajectories and fixed detector measurements can be easily incorporated in the algorithm. However we need to define a driver attribute $I$ and to know how it evolves on the computational domain. That is the tough point because 


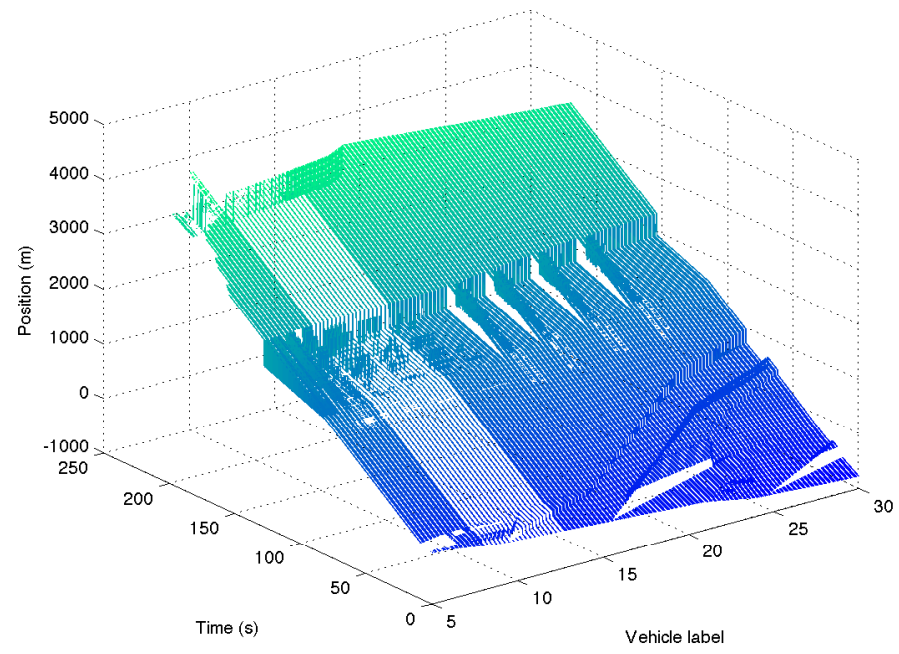

Figure 15: Numerical solution for GSOM PDE obtained on the computation domain $\left[N_{0}, N_{\max }\right] \times\left[t_{0}, t_{\max }\right]$.

it is hard to measure or to evaluate. Notice that an example of speed data assimilation with the ARZ model which is a model of the GSOM family can be found in [38].

\section{Discussion and conclusion}

In this paper, we are interested in the assimilation of different kinds of data into a generic class of macroscopic traffic flow models for improving traffic state estimation. The data come from mixed sources including Lagrangian vehicles trajectories and Eulerian cumulative vehicles counts obtained from fixed detectors. We then consider the models in the Lagrangian system of coordinates which seems to be the most adapted framework for dealing with such data. We describe a computational method providing solutions to macroscopic traffic flow models of the Generic Second Order Modeling (GSOM) family under piecewise affine initial and boundary conditions. The numerical method is based on the variational theory which has been extended to the GSOM family very recently [34. Solution can be computed thanks to Lax-Hopf like formula and a generalization of the inf-morphism property [6]. It is possible to considerably reduce the number of integral curves by following only the characteristics which are the optimal trajectories. Another computational trick is to solve separately many partial problems (by discretizing initial and boundary conditions) instead of the general problem.

Extensions of this work could include more general assumptions on the dynamics $\varphi \neq 0$ of drivers attribute, always beyond piecewise affine value conditions. The computational benefits of the variational method over the finite difference method which is also presented in the paper need to be illustrated on several comparisons. We expect that the variational method has a lower computational cost and a globally higher accuracy compared the other method.

A still open but interesting question is the application of the algorithm to data assimilation on road networks. The challenging point is to deal with fundamental diagrams which will depend on the drivers attribute but also on the spatial position. To our best knowledge LaxHopf formula is not available in that framework because Legendre-Fenchel transform does not apply for space dependent Hamiltonians. One considered possibility is to use the extended concepts of supply and demand functions to models of the GSOM family [37]. This subject is 
being currently investigated by the authors.

\section{Aknowledgements}

The first author would like to thank Jia Li, PhD candidate at UC Davis, California, USA, for deeper insight of his work.

This work was partially supported by the ANR (Agence Nationale de la Recherche) through HJnet project ANR-12-BS01-0008-01.

\section{References}

[1] A. Aw And M. Rascle, Resurrection of "Second Order" Models of traffic flow, SIAM J. Appl. Math. 60 (2000), pp. 916-938. 3

[2] J.P. Aubin, A.M. Bayen, P. Saint-Pierre, Dirichlet problems for some Hamilton-Jacobi equations with inequality constraints, SIAM Journal on Control and Optimization, 47 (2008), pp. 2348-2380. 8 13

[3] J.P. Aubin, A.M. Bayen, P. Saint-Pierre, Viability theory: new directions, Springer, 2011. 8,13

[4] P. Bagnerini, M. Rascle, A multiclass homogenized hyperbolic model of traffic flow, SIAM journal on mathematical analysis, 35 (2003), pp. 949-973. 3

[5] Y. Brenier, Un algorithme rapide pour le calcul de transformées de Legendre-Fenchel discrètes, Comptes rendus de l'Acadmie des sciences. Série 1, Mathématique, 308 (1989), pp. 587-589. 17

[6] C. Claudel, A. Bayen, Lax-Hopf based incorporation of internal boundary conditions into HamiltonJacobi equation. Part I: Theory, IEEE Transactions on Automatic Control. 55(5), (2010), pp. 1142-1157. 8, 13,23

[7] C. Claudel, A. Bayen, Lax-Hopf based incorporation of internal boundary conditions into HamiltonJacobi equation. Part II: Computational methods, IEEE Transactions on Automatic Control. 55(5), (2010), pp. 1158-1174. 8, 11, 12, 13

[8] C. Claudel, A. Bayen, Convex formulations of data assimilation problems for a class of HamiltonJacobi equations, SIAM Journal on Optimization and Control. (2011), pp. 383-402. 8

[9] R.M. Colombo, Hyperbolic phase transitions in traffic flow, SIAM Journal on Applied Mathematics, 63 (2003), pp. 708-721. 4

[10] L. Corrias, Fast Legendre-Fenchel transform and applications to Hamilton-Jacobi equations and conservation laws, SIAM journal on numerical analysis, 33 (1996), pp. 1534-1558. 17

[11] G. Costeseque, J.P. Lebacque, R. Monneau, A convergent scheme for Hamilton-Jacobi equations on a junction: application to traffic, arXiv preprint arXiv:1306.0329 (2013). 12

[12] C.F. Daganzo, The cell transmission model, part II: network traffic, Transportation Research Part B: Methodological, 29 (1995), pp. 79-93. 7

[13] C.F. Daganzo, A variational formulation of kinematic waves: Basic theory and complex boundary conditions, Transportation Research 39B (2005) pp. 187-196. 4, 5, 6, 8

[14] C.F. Daganzo, A variational formulation of kinematic waves: Solution method, Transportation Research 39B (2005) pp. 934-950. 4, 5, 6, 8

[15] C.F. Daganzo, M. Menendez, A variational formulation of kinematic waves: Bottleneck properties and examples, In H.S. Mahmassani, editor, Proceedings of the 16th International Symposium on the Transportation and Traffic Theory, College Park, Maryland, USA, Elsevier, Oxford, 2005, pp. 345-364. 4. 8

[16] C.F. Daganzo, On the variational theory of traffic flow: well-posedness, duality and applications, Networks and Heterogeneous Media, AIMS, 1 (2006), pp. 601-619. 4

[17] A. Duret, C. Buisson, N. Chiabaut, Estimating individual speed-spacing relationship and assessing ability of Newell's car-following model to reproduce trajectories, Transportation Research Record: Journal of the Transportation Research Board, 2088 (2008), pp. 188-197. 9

[18] L.C. Evans, Partial Differential Equations: Second Edition, Graduate Studies in Mathematics 19, American Mathematical Society (2010). 5

[19] M. Garavello And B. Piccoli, Traffic flow on networks, vol.1 of AIMS Series on Applied Mathematics, American Institute of Mathematical Sciences (AIMS), Springfield, MO, (2006) 11 3 
[20] K. Han, T. YAO, T.L. Friesz, Lagrangian-based hydrodynamic model: Freeway traffic estimation, submitted to Transportation Research Part B (2012). 6, 8 , 9

[21] V. Henn, A wave-based resolution scheme for the hydrodynamic LWR traffic flow model, In S.P. Hoogendoorn, S. Luding, P.H.L. Bovy, M. Schreckenberg, and D.E. Wolf, eds., Traffic and Granular Flow03, Springer Berlin Heidelber (2005) pp. 105-124. 7

[22] J.C. Herrera, A.M. BAyen, Incorporation of Lagrangian measurements in freeway traffic state estimation, Transportation Research Part B: Methodological, 44 (2010), pp. 460-481. 2

[23] J.C. Herrera, D.B. Work, R. Herring, X.J. Ban, Q. Jacobson, A.M. Bayen, Evaluation of traffic data obtained via GPS-enabled mobile phones: The Mobile Century field experiment, Transportation Research Part C: Emerging Technologies, 18 (2010), pp. 568-583. 2

[24] M. Herty, C. Kirchner, S. Moutari, M. Rascle, Multicommodity flows on road networks, Communications in Mathematical Sciences, 6 (2008), pp. 171-187. 3

[25] H. Holden, N.H. Risebro, Front tracking for hyperbolic conservation laws, Applied Mathematics Sciences Vol. 152. Springer, 2011. 7

[26] W.L. Jin, H.M. Zhang, Multicommodity kinematic wave simulation model for network traffic flow, Transportation Research Record: Journal of the Transportation Research Board, 1883 (2004), pp. 5967. 3

[27] A. Klar, J.M. Greenberg, M. Rascle, Congestion on multilane highways, SIAM Journal on Applied Mathematics, 63 (2003), pp. 818-833. 3

[28] M.M. Khoshyaran, J.P.Lebacque, A stochastic macroscopic traffic model devoid of diffusion, In Traffic and Granular Flow07, Springer Berlin Heidelberg (2009). 4

[29] J.A. Laval, L. LeClerCQ, The Hamilton-Jacobi partial differential equation and the three representations of traffic flow, Transportation Research Part B, 52 (2013), pp. 17-30. 4

[30] J.P. Lebacque, Les modèles macroscopiques du trafic, Annales des Ponts 67, 1993, pp. 28-45. 10 3, 7

[31] J.P. LeBACQUe, The Godunov scheme and what it means for first order traffic flow models, In J.B. Lesort, editor, 13th ISTTT Symposium, Elsevier, New York, 1996, pp. 647-678. 36

[32] J.P. Lebacque, A two phase extension of the LWR model based on the boundedness of traffic acceleration, In: Michael A.P. Taylor (Eds) Transportation and Traffic Theory in the 21st Century, Proceedings of the 15th International Symposium on Transportation and Traffic Theory, 2002. 3

[33] J.P. LEBACQUE, Two-phase bounded-acceleration traffic flow model: analytical solutions and applications, Transportation Research Record: Journal of the Transportation Research Board, 1852 (2003), pp. 220-230. 3

[34] J.P. Lebacque, M.M. Khoshyaran, A variationnal formulation for higher order macroscopic traffic flow model of the GSOM family, Procedia-Social and Behavioral Sciences, 80 (2013), pp. 370-394. 2 4 9. 10, 12, 19, 23,

[35] J.P. Lebacque, H. Haj-Salem, S. Mammar, Second order traffic flow modeling: supply-demand analysis of the inhomogeneous Riemann problem and of boundary conditions, Proceedings of the 10th Euro Working Group on Transportation (EWGT), 2005. 3

[36] J.P. Lebacque, S. Mammar, H. Haj-Salem, Generic second order traffic flow modelling, In: Allsop, R.E., Bell, M.G.H., Heydecker, B.G. (Eds) Proceedings of the 17th International Symposium on Transportation and Traffic Theory. London: Elsevier, 2007, pp. 755-776. 1, 3, 4

[37] J.P. Lebacque, S. Mammar, H. HaJ-Salem, An intersection model based on the GSOM model, Proceedings of the 17th World Congress, The International Federation of Automatic Control, Seoul, Korea, (2008), pp. 7148-7153. 23

[38] J.P. Lebacque, S. Mammar, H. Haj-Salem, Riemann problem resolution and Godunov scheme for the Aw-Rascle-Zhang model, Transportation Science, 43 (2009), pp. 531-545. 23

[39] L. LeClercQ, Bounded acceleration close to fixed and moving bottlenecks, Transportation Research Part B: Methodological, 41 (2007), pp. 309-319. 3

[40] L. LeclercQ, J. Laval, E. Chevallier, The Lagrangian coordinates and what it means for first order traffic flow models, In: Allsop, R.E., Bell, M.G.H., Heydecker, B.G. (Eds) Proceedings of the 17th International Symposium on Transportation and Traffic Theory. London: Elsevier, 2007, p. 735-753. 6

[41] J. Li, H.M. Zhang, The Variational Formulation of a Non-equilibrium Traffic Flow Model: Theory and Implications, Procedia-Social and Behavioral Sciences, 80 (2013), pp. 327-340. 9] 11

[42] M.J. Lighthill, G.B. Whitham, On kinetic waves. II. Theory of Traffic Flows on Long Crowded Roads, Proc. Roy. Soc. London Ser. A, 229 (1955), pp. 317-345. 13

[43] Y. LuCET, Faster than the fast Legendre transform, the linear-time Legendre transform, Numerical Algorithms, 16 (1997), pp. 171-185. 17 
[44] Y. Makigami, G.F. Newell, R. Rothery, Three-dimensional representation of traffic flow, Transportation Science, 5 (1971), pp. 302-313. 4

[45] P.E. Mazaré, A.H. Dehwah, C.G. Claudel, A.M. Bayen, Analytical and grid-free solutions to the LighthillWhithamRichards traffic flow model, Transportation Research Part B, 45 (2011), pp. 1727-1748. 8. 11

[46] K. Moskowitz, L. Newan, Notes on freeway capacity, Highway Research Record, 1963. 4

[47] G.F. Newell, A simplified theory of kinematic waves in highway traffic, (I) General theory, (II) Queueing at freeway bottlenecks, (III) Multi-destination flows, Transportation Research B, 27 (1993), pp. 281-313. 4, 6

[48] G.F. Newell, A simplified car-following theory: a lower order model, Transportation Research Part B: Methodological, 36 (2002), pp. 195-205. 7

[49] B. Piccoli, K. Han, T.L. Friesz, T. Yao, Second Order Models and Traffic Data from Mobile Phones, arXiv preprint arXiv:1211.0319 (2012). 2

[50] S. Qiu, M. Abdelaziz, F. Abdellatif, C.G. Claudel, Exact and grid-free solutions to the LighthillWhithamRichards traffic flow model with bounded acceleration for a class of fundamental diagrams, Transportation Research Part B: Methodological, 55 (2013), pp. 282-306.

[51] P. I. Richards, Shock Waves on the Highway, Oper. Res., 4 (1956), pp. 42-51. 1, 3

[52] D.B. Work, O.P. Tossavainen, Q. Jacobson, A.M. BAyen, Lagrangian sensing: traffic estimation with mobile devices, American Control Conference 2009, IEEE, pp. 1536-1543. 2

[53] H.M. Zhang, A non-equilibrium traffic model devoid of gas like behavior, Transportation Research Part B, 36, no. 3, 2002, pp. 275-290. 1] 3

[54] http://traffic.berkeley.edu/ 2 , 9 


\section{Contents}

1 Introduction 1

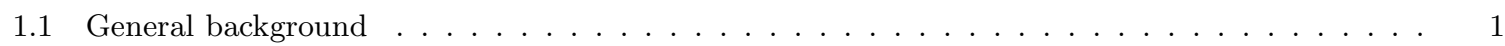

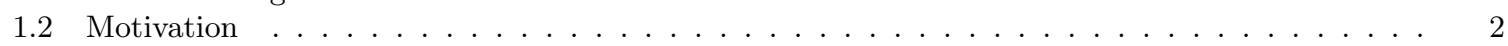

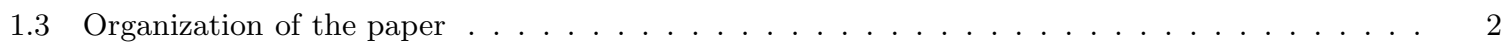

2 GSOM traffic flow models 3

2.1 Formulation of GSOM models $\ldots \ldots \ldots \ldots \ldots \ldots \ldots \ldots$

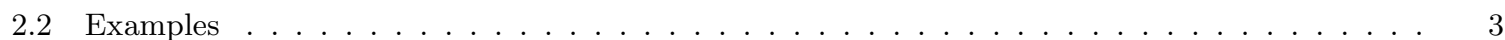

3 Variational principles in traffic flow modeling 4

3.1 Variational formulation of the LWR model . . . . . . . . . . . . . . . . . . . . . . . . . . . 4

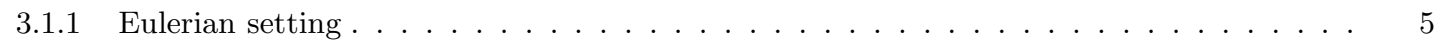

3.1 .2 Lagrangian setting . . . . . . . . . . . . . . . . . . . . . . . . . 6

3.2 Computational aspects for the LWR model $\ldots \ldots \ldots \ldots \ldots \ldots \ldots$

3.2 .1 Motivation of Lax-Hopf algorithms $\ldots \ldots \ldots \ldots \ldots \ldots \ldots$

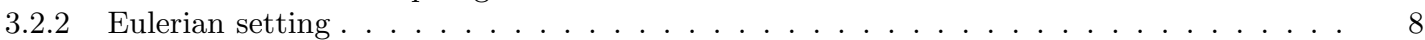

3.2 .3 Lagrangian setting . . . . . . . . . . . . . . . . . . . . . . . . . . . 8

3.3 Variational formulation of the GSOM family $\ldots \ldots \ldots \ldots \ldots \ldots \ldots \ldots \ldots \ldots$

$3.3 .1 \quad$ Eulerian setting. . . . . . . . . . . . . . . . . . . . . . . . . . . . . 9

3.3 .2 Lagrangian setting . . . . . . . . . . . . . . . . . . . . . . . . . . . . . . 10

3.4 Review of computational methods for GSOM models $\ldots \ldots \ldots \ldots \ldots \ldots \ldots$

$\begin{array}{|lr|}4 \text { Computational aspects } & 11\end{array}$

$4.1 \quad$ A finite difference scheme . . . . . . . . . . . . . . . . . . . . . . . . . . . . . . . . . . 12

$4.2 \quad$ A "grid free" scheme . . . . . . . . . . . . . . . . . . . . . . . . . . . . . . . . . 12

4.3 Algorithm for piecewise affine value conditions $\ldots \ldots \ldots \ldots \ldots \ldots \ldots$

$4.3 .1 \quad$ Initial conditions . . . . . . . . . . . . . . . . . . . . . . . . . . . 14

4.3 .2 "Upstream" boundarv conditions . . . . . . . . . . . . . . . . . . . . . . 17

4.3 .3 Internal boundarv conditions $\ldots \ldots \ldots \ldots \ldots \ldots \ldots$

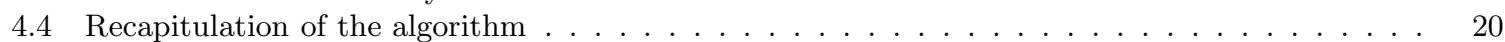

$\begin{array}{lll}5 & \text { Numerical example } & 21\end{array}$

6 Discussion and conclusion 23 